Published in final edited form as:

Nat Immunol. 2018 September ; 19(9): 954-962. doi:10.1038/s41590-018-0185-0.

\title{
Endogenous glucocorticoids control host resistance to viral infection through the tissue-specific regulation of PD-1 expression on NK cells
}

\author{
Linda Quatrini $^{1}$, Elisabeth Wieduwild ${ }^{1}$, Bertrand Escaliere ${ }^{1}$, Jessica Filtjens ${ }^{1}$, Lionel \\ Chasson ${ }^{1}$, Caroline Laprie ${ }^{1}$, Eric Vivier ${ }^{1,2,3}$, and Sophie Ugolini ${ }^{1,}{ }^{*}$ \\ ${ }^{1}$ Aix Marseille Univ, CNRS, INSERM, CIML, Centre d'Immunologie de Marseille-Luminy, \\ Marseille, France \\ ${ }^{2}$ Innate Pharma Research Laboratories, Innate Pharma, Marseille, France \\ ${ }^{3}$ Service d'Immunologie, Marseille Immunopole, Hôpital de la Timone, Assistance Publique des \\ Hôpitaux de Marseille
}

\begin{abstract}
Controlling the balance between immunity and immunopathology is crucial for host resistance to pathogens. After infection, activation of the hypothalamic-pituitary-adrenal (HPA) axis leads to the production of glucocorticoids. However, the pleiotropic effects of these steroid hormones make it difficult to delineate their precise role(s) in vivo. Here we found that the regulation of natural killer (NK) cell function by the glucocorticoid receptor (GR) was required for host survival to mouse cytomegalovirus infection. Mechanistically, endogenous glucocorticoids produced shortly after infection induced the selective and tissue-specific expression of the checkpoint molecule PD-1 on NK cells. This glucocorticoid-PD-1 pathway limited the production of the cytokine interferon- $\gamma($ IFN- $\gamma$ ) by splenic NK cells, which prevented lethal immunopathology. Importantly, this regulation did not compromise viral clearance. Thus, the fine-tuning of NK cell functions by the HPA axis preserved tissue integrity without impairing pathogen elimination, which reveals a novel aspect of neuroimmune regulation.
\end{abstract}

The immune system protects the host organism against infectious diseases, principally by eradicating infectious agents. However, pathogen elimination frequently entails collateral

Users may view, print, copy, and download text and data-mine the content in such documents, for the purposes of academic research, subject always to the full Conditions of use:http://www.nature.com/authors/editorial_policies/license.html\#terms

*Correspondence: ugolini@ciml.univ-mrs.fr.

Data availability

The data that support the findings of this study are available from the corresponding author upon request. The RNA sequencing data have been deposited in the GEO repository under accession code GSE114827.

Author contributions

LQ designed and performed experiments and analyzed data. EW and JF performed experiments. BE performed RNA-seq analysis. LC processed the histology samples and CL performed their expert pathological examinations. EV provided the $N c r 1$ re mouse model and important advice during the manuscript preparation. SU conceived, designed and directed the study. LQ and SU wrote the manuscript. All authors reviewed and provided input on the manuscript.

Competing financial interests

$\mathrm{EV}$ is a cofounder and employee of Innate Pharma. The other authors declare no competing financial interests. 
tissue damage and inflammation, potentially decreasing host fitness 1 . Neuroendocrineimmune interactions play an important role in these regulatory processes, but the mechanisms involved remain unclear 2, 3. Endogenous glucocorticoids (cortisol in humans and corticosterone in rodents) are essential steroid hormones that regulate many physiological and developmental processes. They are synthesized in the adrenal cortex upon activation of the hypothalamic-pituitary-adrenal (HPA) axis, a neural-endocrine core for coordinating physiological responses to external stimuli 4. Glucocorticoids released into the blood diffuse through cell membranes to bind cytosolic glucocorticoid receptor (GR), which is ubiquitously expressed and induces changes in gene expression 5. The HPA neuroendocrine pathway restores homeostasis by limiting and resolving inflammation in many conditions 2. In models of systemic inflammation, such as lipopolysaccharide (LPS)induced sepsis, glucocorticoids specifically inhibit pro-inflammatory cytokine production in several immune cell-types $6,7,8,9$.

The HPA axis is also activated during infection with viruses, such as influenza 10 and cytomegalovirus (CMV) 11. Murine CMV (MCMV) is a beta herpesvirus used as a model for systemic human CMV infection, which can cause severe disease, especially in immunocompromised patients. Mice rendered glucocorticoid-deficient by adrenalectomy are more susceptible to MCMV-induced death, due to the deleterious effects of cytokineinduced over-inflammation, and resistance is restored by corticosterone replacement 12 . Finally, glucocorticoids are clinically effective against many inflammatory and autoimmune diseases 13. In all these conditions, the underlying mechanisms of glucocorticoids remain unclear, because GRs are expressed by many hematopoietic and non-hematopoietic cells and can impact on multiple signaling pathways.

During acute infection, the cytokine interferon- $\gamma$ (IFN- $\gamma$ ), produced by innate lymphoid cells (ILCs) is essential for antiviral defense $14,15,16$. These IFN- $\gamma$-producing ILCs include spleen and liver natural killer (NK) cells and liver ILC1s, which all express the GR 6. We investigated here the role of the GR in ILC subsets of relevance during MCMV infection using mice with a conditional deletion of the GR gene in ILCs expressing NCR1, which include NK cells, ILC1s and a subset of ILC3s. We found that endogenous glucocorticoids produced rapidly after infection induced the selective and tissue-specific expression of the immune checkpoint PD-1 on the surface of spleen NK cells. Concomitantly, PD1 ligands were upregulated in several immune cell subsets. PD-1 signalling was required for host survival to infection and acted by limiting the production of IFN- $\gamma$ by NK in the spleen which prevented immunopathology. This neuroendocrineimmune axis controlled immune tolerance but did not impair viral clearance. Our findings therefore demonstrate a major role for the HPA axis in promoting host resistance to an infectious disease through regulation of the PD-1 inhibitory pathway in an ILC subset.

\section{Results}

\section{The regulation of NK cell functions by glucocorticoids is organ-specific}

During acute infection, MCMV has a high tropism for the spleen and the liver 17. In these organs, NK cells are required for host resistance to infection, and they mediate their antiviral function through cytokine- and killing-dependent mechanisms 18. Consistent with 
previous findings 11, 19, MCMV infection in C57BL/6J wild-type mice induced corticosterone release into the bloodstream, with a peak at $36 \mathrm{~h}$ post-infection (Fig. 1a), suggesting that the HPA axis may play a role during the early phase of the infection when NK cells are activated 14 . We first analyzed the effect of glucocorticoids on the cytotoxic activity of spleen $\mathrm{CD} 3{ }^{-} \mathrm{NCR} 1^{+} \mathrm{NK}$ cells in vitro by stimulating splenocytes isolated from C57BL/6J wild-type mice with antibodies against NK1.1 or PMA and ionomycin.

Corticosterone treatment had no effect on degranulation of NK cells, as assessed by measuring CD107a expression (Supplementary Fig. 1a). We then analyzed the role of the GR pathway in NCR1 ${ }^{+}$ILCs in vivo using $N$ cr $1^{\text {iCre/ } /+} \mathrm{Nr} 3 \mathrm{c} 1^{\text {LoxP/LoxP }}$ mice (hereafter $\mathrm{GR}^{N c r 1-\mathrm{iCre}}$ mice), in which the gene encoding GR $(\mathrm{N} r 3 c 1)$ is specifically deleted in NCR $1^{+}$ cells which express the Cre recombinase 6, 20,21. Sex matched $\mathrm{Ncr}^{\mathrm{iCr} /+}$ littermates in which the Cre recombinase is expressed in NCR $1^{+}$cells, but the $\mathrm{Nr} 3 \mathrm{cr} 1$ gene is not deleted, were used throughout as controls (referred to hereafter as $\mathrm{WT}^{N c r 1-\mathrm{iCre}}$ ), unless otherwise specified. In steady-state conditions, NCR $1^{+}$ILCs in $\mathrm{GR}^{N c r 1-\mathrm{iCre}}$ mice (i.e. NK cells, ILC1s and a subset of ILC3s) show normal development, maturation and functions 6 . We infected $\mathrm{GR}^{N c r l-\mathrm{iCre}}$ and $\mathrm{WT}^{N c r 1-\mathrm{iCre}}$ littermates with $\mathrm{MCMV}$, and their immune responses were analyzed at $44 \mathrm{~h}$ post-infection, after blood corticosterone concentration had peaked. The concentrations of corticosterone in the serum were similar in $\mathrm{GR}^{N c r l-\mathrm{iCre}}$ and $\mathrm{WT}^{N c r 1-\mathrm{iCre}}$ control mice (Supplementary Fig. 1b). Granzyme B (GrzB), a surrogate marker of NK cell cytotoxicity, was similarly upregulated in $\mathrm{NK} 1.1^{+} \mathrm{NCR} 1^{+} \mathrm{DX} 5^{+} \mathrm{CD} 49 \mathrm{a}^{-} \mathrm{NK}$ cells from $\mathrm{GR}^{N c r 1-\mathrm{iCre}}$ and $\mathrm{WT}^{N c r 1-\mathrm{iCre}}$ mice, both in spleen and in liver (Supplementary Fig. 1c). In contrast, expression of IFN- $\gamma$ was higher in spleen GR $^{N c r 1-\text { iCre }}$ NK cells compared to $\mathrm{WT}^{\text {Ncr } 1 \text {-iCre }}$ control NK cells, as measured by mean fluorescence intensity and in terms of the percentage of IFN- $\gamma^{+}$cells (Fig. $1 \mathrm{~b}$ and Supplementary Fig. 1d). Although corticosterone is known to have a systemic distribution, liver $\mathrm{GR}^{N c r 1 \text {-iCre }}$ and $\mathrm{WT}^{N c r 1 \text {-iCre }}$ $\mathrm{NCR}^{+} \mathrm{NK}$ cells and ILC1s had similar expression of IFN- $\gamma$ (Fig. 1c, d), suggesting the effect of glucocorticoids was tissue-specific.

Histological analysis of the spleen indicated that $\mathrm{GR}^{N c r l-\mathrm{iCre}}$ mice had more marked lesions, associated with coalescing and granulomatous necrotic splenitis in the marginal zone compared to WT ${ }^{N c r 1-i C r e}$ control mice (Fig. 2a, b). In contrast, no significant difference in the nature or extent of the liver lesions was observed between $\mathrm{GR}^{N c r} 1$-iCre and control mice, with mild multifocal pyogranulomatous and necrotic hepatitis in both genotypes (Fig. 2c, d). The viral load in the spleen and the liver was similar in $\mathrm{GR}^{N c r 1-\mathrm{iCre}}$ and $\mathrm{WT}^{N c r 1-\mathrm{iCre}}$ mice (Fig. 2e), suggesting that the inflammation-induced tissue damage was not due to an impairment of viral clearance.

NCR $1^{+}$ILC3s are known to express GR and the GR encoding gene is also deleted in NCR1 ${ }^{+}$ ILC3s from $\mathrm{GR}^{N c r 1-\mathrm{iCre}}$ mice 6 . These ILC3s are mostly resident in mucosal tissues, where they produce interleukin 22 (IL-22) and IL-17, but not IFN- $\gamma 22$, and as such they are thought to have minimal involvement in the immune response against MCMV. Nonetheless, we assessed the effect of endogenous glucocorticoids on these cells upon MCMV infection. MCMV infection did not induce an increase in IL-22 and IL-17 production in NCR1 ${ }^{+}$ILC3 from the small intestine in $\mathrm{WT}^{N c r 1-\mathrm{iCre}}$ and $\mathrm{GR}^{N c r}$-iCre mice (Supplementary Fig. 2a-d). The frequency of NCR $1^{+}$ILC3s in the small intestine was also unaffected (Supplementary Fig. 2e), suggesting NCR1 $1^{+}$ILC3s do not have a major impact on the immune response to 
MCMV infection. Collectively, these results indicate that responsiveness of NCR1 ${ }^{+}$ILCs to glucocorticoids produced during acute MCMV infection controls spleen inflammation and specifically regulates the production of IFN- $\gamma$ by spleen NK cells.

\section{Endogenous glucocorticoids induce PD-1 expression on splenic NK cells}

Next, we performed RNA-sequencing (RNA-Seq) on spleen $\mathrm{CD}^{-} \mathrm{CD}^{-} 9^{-}$

$\mathrm{NK} 1.1^{+} \mathrm{NCR} 1^{+} \mathrm{DX} 5^{+} \mathrm{NK}$ cells, liver CD3 ${ }^{-} \mathrm{CD} 19^{-} \mathrm{NK} 1.1^{+} \mathrm{NCR} 1^{+} \mathrm{DX} 5^{+} \mathrm{CD} 49 \mathrm{a}^{-} \mathrm{NK}$ cells and liver CD3-CD19-NK1.1 ${ }^{+} \mathrm{NCR} 1^{+} \mathrm{DX} 5^{-} \mathrm{CD} 49 \mathrm{a}^{+} \mathrm{ILC} 1 \mathrm{~s}$ sorted from $\mathrm{GR}^{N c r 1-\mathrm{iCre}}$ and $\mathrm{WT}^{N c r 1-\text {-iCre }}$ control mice $44 \mathrm{~h}$ post-infection (Supplementary Fig. 3). A pairwise comparison of gene expression between the two genotypes for spleen NK cells, liver NK cells and liver ILC1s identified differentially expressed genes for each ILC subset (Supplementary Table 1). Unsupervised genome-wide analysis indicated that the GR-dependent transcriptomic changes differed between spleen NK cells, liver NK cells and liver ILC1s (Supplementary Table 1), suggesting that the regulation of gene expression by glucocorticoids was both organ- and cell lineage-specific in vivo. To identify candidate genes associated with the selective hyperinflammation observed in the spleen of $\mathrm{GR}^{N c r 1-\mathrm{iCre}}$ mice, we focused on genes that were differentially expressed between $\mathrm{WT}^{N c r 1 \text {-iCre }}$ control and $\mathrm{GR}^{N c r l-\mathrm{iCre}}$ spleen NK cells (Fig. 3a). In addition to $N r 3 c 1$, which encodes GR, the expression of $T s c 22 d 3$, which encodes the glucocorticoid-induced protein GILZ and the expression of Pdcd1, which encodes the receptor PD-1 were significantly downregulated in spleen NK cells from $\mathrm{GR}^{N c r 1-\mathrm{iCre}}$ versus $\mathrm{WT}^{N c r 1-\mathrm{iCre}}$ littermates after infection (Fig. 3a and Supplementary Table $1)$.

Consistent with the rapid and ubiquitous induction of $T s c 22 d 3$ by glucocorticoids in many cell types and inflammatory conditions 23 , differential expression of $T s c 22 d 3$ was seen in both spleen and liver NK cells from $\mathrm{GR}^{N c r l \text {-iCre }}$ versus $\mathrm{WT}^{N^{N} 1 \text { - } \text {-iCre }}$ mice upon infection (Supplementary Table 1). GILZ is a mediator of the anti-inflammatory effects of glucocorticoids and regulates survival, homeostasis and apoptosis in various cell types, including lymphoid cells 24,25 . We measured NK cell proliferation and apoptosis in the spleen and liver of $\mathrm{GR}^{\text {Ncr } 1 \text {-iCre }}$ mice and littermate controls $44 \mathrm{~h}$ after infection using Ki67 and AnnexinV/PI flow cytometry staining, respectively. Despite the downregulation of $T s c 22 d 3$ in spleen and liver $\mathrm{GR}^{N c r 1-\text { iCre }}$ NK cells, there was no difference in their level of proliferation and apoptosis compared to WT ${ }^{N c r 1-\text { iCre }}$ NK cells (Supplementary Fig. 4 a,b). Liver ILC1 homeostasis was also similar in $\mathrm{WT}^{N c r 1-\mathrm{iCre}}$ and $\mathrm{GR}^{N c r l-\text { iCre }}$ mice

(Supplementary Fig. 4c), suggesting that GILZ does not play a major role in the selective

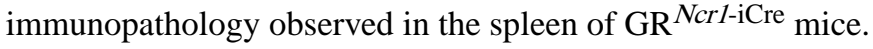

In contrast to $T s c 22 d 3$, the differential expression of $P d c d 1$ was selectively observed in spleen but not liver NK cells from MCMV-infected GR ${ }^{N c r 1-i C r e}$ mice (Supplementary Table 1). In addition, $P d c d 1$ expression was similar in liver ILC1s from MCMV-infected $\mathrm{GR}^{\text {Ncr } 1 \text {-iCre }}$ and $\mathrm{WT}^{N c r 1-\mathrm{iCre}}$ mice. PD- 1 is an inhibitory cell-surface receptor that downregulates T cell activity 26. PD-1 protein was not expressed on the surface of spleen and liver NK cells from uninfected $\mathrm{GR}^{N c r 1-\mathrm{iCre}}$ and $\mathrm{WT}^{N c r 1-\mathrm{iCre}}$ mice, but was detected on splenic NK cells from MCMV-infected WT ${ }^{N c r 1 \text {-iCre }}$ control mice $44 \mathrm{~h}$ post-infection (Fig. $3 b)$. Importantly, we could not detect PD-1 in spleen NK cells from MCMV-infected 
$\mathrm{GR}^{\text {Ncr } 1 \text {-iCre }}$ mice (Fig. 3b), showing induction of PD-1 was dependent on the glucocorticoidGR pathway. PD-1 expression was not detected on spleen macrophages, neutrophils or dendritic cells (DCs) in either non-infected and MCMV-infected wild-type mice 44h postinfection (Supplementary Fig. 5). In the spleen of non-infected $\mathrm{WT}^{N c r l-\text { iCre }}$ and $\mathrm{GR}^{N c r l \text {-iCre }}$ mice, a discrete subset of $\mathrm{T}$ cells (less than 4\%) had low basal expression of PD-1, but this $\mathrm{T}$ cell subset did not expand and PD-1 expression on T cells did not change upon MCMV infection in either genotypes (Fig. 3c). Expression of PD-1 was not detected on liver NK cells and ILC1 (Fig. 3d, e) consistent with the transcriptomic data. Similar results were obtained using two different clones of anti-PD-1 antibodies (J43 and RMP1-30) (Fig. 3b). These data indicate that glucocorticoids released upon MCMV infection induce the GRdependent de-novo expression of PD-1 on spleen NK cells by regulating Pdcd1 gene transcription.

\section{NK cell PD-1 expression depends on the cytokine microenvironment}

Liver NK cells expressed GR both at steady-state 6 and upon MCMV infection (Supplementary Fig. 6a). Tsc22d3 expression was downregulated similarly in spleen and liver $\mathrm{GR}^{N c r 1 \text {-iCre }} \mathrm{NK}$ cells compared to corresponding $\mathrm{WT}^{N c r 1 \text {-iCre }}$ control cells(Supplementary Table 1), indicating liver NK cells were responsive to GR signaling. Because the differential expression of PD-1 on liver and spleen NK cells could not be explained by an impaired responsiveness to glucocorticoids, we tested whether it was dependent on the presence of tissue-specific inflammatory cytokines in the microenvironment. We focused on the main cytokines known to modulate NK cell activation. First, RNA from spleen and liver tissue homogenates were extracted from MCMV-infected (44h post-infection) and non-infected wild-type mice and the expression of mRNA for $I 112,1115$ and $I 118$ was assessed by RT-qPCR. We did not detect any significant difference in 1118 mRNA expression before and after infection in either organs (Supplementary Fig. 6b). In contrast, expression of $I 112$ mRNA was up-regulated by infection to a greater extent in the liver compared to the spleen, and 1115 mRNA expression was increased exclusively in the spleen of infected mice compared to un-infected (Fig. 4a). Similar expression patterns for $I 112, I 115$ and $I 118$ were observed in $\mathrm{WT}^{N c r I-\mathrm{iCre}}$ and $\mathrm{GR}^{N c r 1 \text {-iCre }}$ mice (data not shown). These data suggested that the cytokine microenvironment differed between liver and spleen during MCMV infection.

Next we tested whether different combinations of cytokines in the medium modified the effect of GR activation on NK cells in vitro. Splenocytes from WT ${ }^{N c r 1-i C r e}$ and $\mathrm{GR}^{N c r 1-\mathrm{iCre}}$ mice were stimulated for $48 \mathrm{~h}$ with different combinations of IL-12, IL-15 and IL-18 in the presence or absence of corticosterone. In the absence of corticosterone, PD-1 expression was not induced on $\mathrm{CD}^{-}{ }^{-} \mathrm{NCR} 1^{+} \mathrm{NK} 1.1^{+} \mathrm{NK}$ cells or $\mathrm{CD}^{+} \mathrm{T}$ cells (Fig. $4 \mathrm{~b}$ and data not shown). Similarly, corticosterone, alone or in combination with IL-15 or with IL-12 and IL-15, did not induce the expression of PD-1 on NK cells or T cells from either genotypes (Fig. 4b and data not shown). In contrast, treatment with corticosterone, IL-15 and IL-18 induced PD-1 expression on NK cells from WT ${ }^{N c r 1 \text {-iCre }}$ control but not from $\mathrm{GR}^{N c r 1 \text {-iCre }}$ mice in a dosedependent manner (Fig. $4 \mathrm{~b}$ and c). This treatment did not induce the expression of PD-1 on $\mathrm{T}$ cells from $\mathrm{WT}^{N c r 1-\mathrm{iCre}}$ or $\mathrm{GR}^{N c r 1-\mathrm{iCre}}$ mice (Fig. $4 \mathrm{~b}$ and data not shown). Addition of IL-12 abolished the corticosterone, IL-15 and IL-18-dependent induction of PD-1 in 
$\mathrm{WT}^{\text {Ncrl-iCre }}$ control NK cells (Fig. 4b). Similar results were obtained when stimulating $\mathrm{NK} 1.1^{+} \mathrm{NCR} 1^{+} \mathrm{NK}$ cells enriched from splenocytes (86-89\% of purity) (Fig. $4 \mathrm{~d}$ and data not shown), suggesting the effect of IL-15 and IL-18 on NK cells was cell-intrinsic. These data indicated that GR induction of PD-1 was cell type-specific and dependent on NK cell cytokine microenvironment.

\section{The GR-PD-1 axis inhibits IFN- $\gamma$ production in spleen NK cells}

The activating NK cell receptor Ly49H mediates resistance to MCMV infection in C57BL/6J mice, by specifically binding the virally encoded protein m157 27. As such, we investigated the link between expression of PD-1 and Ly49H on spleen NK cells during MCMV infection. Upon infection of wild-type C57BL/6J mice, PD-1 was similarly upregulated on both $\mathrm{Ly}_{49 \mathrm{H}^{+}}$and $\mathrm{Ly}_{49 \mathrm{H}^{-}}$spleen $\mathrm{NK}$ subsets $44 \mathrm{~h}$ post-infection (Supplementary Fig. 7a). Moreover, the frequency of $\mathrm{Ly}_{49 \mathrm{H}^{+}}$spleen NK cells 5 days postinfection was similar in $\mathrm{GR}^{N c r l-\mathrm{iCre}}$ and $\mathrm{WT}^{N c r 1-\mathrm{iCre}}$ control mice (Supplementary Fig. 7b), suggesting GR signaling and expression of PD-1 did not affect the expansion of the m157specific NK cells during MCMV infection.

To further investigate whether PD-1 expression and signaling in spleen NK cells may have a functional relevance, we tested whether the PD-1 ligands PD-L1 and PD-L2 were expressed in wild-type mice during MCMV infection. Cytofluorimetric analysis revealed that PD-L1 expression was upregulated on macrophages, DCs, neutrophils, T, B and NK cells from spleen of MCMV-infected compared to non-infected wild-type mice (Fig. 5a), while PD-L2 was expressed only on DCs upon MCMV infection (Fig. 5b). To test whether the potential engagement of PD-1 by its ligands controlled the function of NK cells in the spleen, MCMV infection of $\mathrm{GR}^{N c r 1-\mathrm{iCre}}$ and $\mathrm{WT}^{N c r 1-\mathrm{iCre}}$ control mice was followed by the administration of PD-1 blocking antibodies (PD-1 Ab) or isotype control antibodies (Ig). Antibodies were injected intraperitoneally 1 day post-infection, before the activation of the HPA axis and glucocorticoid release into the bloodstream. At 44h post-infection, the frequency of IFN- $\gamma^{+}$ splenic NK cells in $\mathrm{WT}^{N c r 1-\mathrm{iCre}}$ mice receiving the PD-1 blockade (about $61 \%$ ) was higher compared to Ig-injected $\mathrm{WT}^{N c r 1-\mathrm{iCre}}$ mice (about $33 \%$ ), but similar to that seen in $\mathrm{GR}^{N c r 1-\mathrm{iCre}}$ mice treated with Ig (54\%) (Fig. 5c). There was no increase in the frequency of IFN- $\gamma^{+}$splenic NK cells in $\mathrm{GR}^{N c r 1-\mathrm{iCre}}$ mice receiving the PD-1 blockade compared to Iginjected mice (Fig. 5c), indicating a strict dependence of the effect of PD-1 on GRexpressing NCR $1^{+} \mathrm{NK}$ cells. Moreover, compared to Ig treatment, PD-1 blockade had no effect on the frequency of IFN- $\gamma^{+}$NK cells in the liver of $\mathrm{WT}^{\text {Ncrl-iCre }}$ mice (Fig. 5d), consistent with the lack of PD-1 expression on liver NK cells. Thus, during MCMV infection, PD-1 signaling in spleen NK cells limits IFN- $\gamma$ production by these cells. This regulation occurs in the spleen, but not in the liver.

\section{GR and PD-1 are required for host resistance to MCMV infection}

To assess the impact of the glucocorticoid-PD1 axis on host resistance to viral infection, we infected 9-10 weeks old GR ${ }^{N c r 1-\mathrm{iCre}}$ and $\mathrm{WT}^{N c r 1 \text {-iCre }}$ control mice with an intraperitoneal median lethal dose (LD50) of MCMV. GR ${ }^{N c r l-i C r e}$ mice had a higher mortality (100\%) compared to control littermates (62\%) (Fig. 6a), indicating that glucocorticoid signaling in NCR1 ${ }^{+}$ILCs was required for host resistance to viral infection. To evaluate the specific 
contribution of PD-1-PD-1 ligand interaction to host resistance, we infected C57BL/6J wildtype female mice with the LD50 of MCMV, followed by the intraperitoneal administration of PD-1 blocking antibodies or isotype control antibodies 1 day post-infection. All mice receiving PD-1 blocking antibodies succumbed to infection within a week (Fig. 6b), resulting in a mortality similar to that of $\mathrm{GR}^{N c r 1 \text {-iCre }}$ mice (Fig. 6a). Importantly, this greater susceptibility to infection was not due to differences in viral replication rates in $\mathrm{GR}^{N c r 1 \text {-iCre }}$ mice (Fig. 6c) or in wild-type mice receiving the PD-1 blocking antibody (Fig. 6d) compared to their respective control mice, suggesting the GR-PD-1 regulatory pathway did not impair viral clearance. Wild-type mice receiving anti-PD-1 antibodies presented more marked immunopathological features in the spleen, with larger areas of coalescing necrotic and granulomatous splenitis in the marginal zone compared to wild-type mice receiving control Ig (Fig. 6e). In contrast, hepatic lesion severity was not affected by PD-1 blockade, with both groups of mice displaying moderate-to-marked necrotic and pyogranulomatous hepatitis, with intranuclear inclusions in hepatocytes (Fig. 6e). MCMV-infected wild-type mice treated with anti-PD-1 antibodies were, therefore, phenocopies of the $\mathrm{GR}^{N c r 1-\mathrm{iCre}}$ mice, indicating PD-1 expression mediated the GR-dependent host protection from infectioninduced immunopathology.

\section{The GR-PD1-IFN- $\gamma$ pathway prevents lethal spleen immunopathology}

PD-1-deficient mice infected with the murine hepatitis virus strain-3 (MHV-3) were reported to have severe spleen and liver damage and high expression of FGL2, a molecule involved in fibrinogen deposition 28. Using RT-qPCR, we found no difference in the expression of $f g l 2$ in spleen tissue homogenates following Ig injection or PD-1 blockade in MCMV-infected C57BL/6J wild-type mice (data not shown). To further investigate the mechanisms involved in the lethal immunopathological condition in the MCMV-infected GR ${ }^{N c r 1-i C r e}$ and PD-1 antibody treated mice, we performed cytometric bead array measurement of inflammatory cytokines concentration in serum, spleen and liver upon infection. Three days post-infection, the amounts of IL-6, TNF and IL-10 in the serum and tissues from MCMV-infected $\mathrm{GR}^{N c r 1-\mathrm{iCre}}$ and PD-1 Ab-treated wild-type mice were similar to those seen in $\mathrm{WT}^{N c r 1 \text {-iCre }}$ and Ig-treated wild-type mice (Supplementary Fig. 8). In contrast, we detected higher IFN- $\gamma$ production in the spleen and the serum, but not in the liver of MCMV-infected $\mathrm{GR}^{N c r l-i C r e}$ and PD-1 Ab-treated wild-type mice compared respectively to $\mathrm{WT}^{N c r 1-\mathrm{iCre}}$ and Ig-treated wild-type mice (Fig. $6 \mathrm{f}$ and g). To test whether the NK cell-derived IFN- $\gamma$ in the spleen of $\mathrm{GR}^{N c r 1-\mathrm{iCre}}$ mice may have a role in spleen hyperinflammation and immunopathology, we treated $\mathrm{WT}^{N c r 1-\mathrm{iCre}}$ and $\mathrm{GR}^{N c r 1-\mathrm{iCre}}$ mice with an IFN- $\gamma$ blocking antibody or an isotype control (IgG1) on day 1 post-infection, at a time when the HPA axis was activated. Histological analysis revealed marked-to-severe inflammation in the spleen of IgG1-treated $\mathrm{GR}^{N c r 1-\mathrm{iCre}}$ mice on day 3 post-infection, with large and coalescing pyogranulomas and necrotic foci throughout the splenic parenchyma (Fig. 7a). We also observed the destruction of the white pulp, including, in particular, the periarteriolar lymphoid sheath (PALS), which displayed decreased cellularity and atrophy in the severely affected areas and partial replacement by necrotic and granulomatous inflammation (Fig. 7a). Morphometric assessment of the PALS indicated IgG1-treated $\mathrm{GR}^{N c r l-\text { iCre }}$ mice displayed a more severe destruction of splenic architecture, with a decreased surface of PALS areas on spleen sections than IgG1-treated $\mathrm{WT}^{N c r 1 \text {-iCre }}$ mice(Fig. 7b). In contrast, $\mathrm{GR}^{N c r 1 \text {-iCre }}$ mice treated 
with the IFN- $\gamma$ neutralizing antibody showed a rescue of spleen immunopathology to a level similar to that of $\mathrm{WT}^{N c r 1-\mathrm{iCre}}$ mice injected with IgG1 or anti-IFN- $\gamma$ antibody (Fig. 7a,b). Thus, the glucocorticoids produced upon MCMV infection controlled IFN- $\gamma$ production by spleen NK cells and IFN $\gamma$-mediated spleen immunopathology.

\section{Discussion}

Here we found that endogenous glucocorticoids produced in response to MCMV infection selectively induced the expression of PD-1 in spleen NK cells, thus controlling their IFN- $\gamma$ production and preventing the development of a lethal immunopathology in the organ. This regulatory pathway was organ-specific and did not affect viral clearance.

Expression of PD-1 could be induced in vitro on NK cells by glucocorticoid treatment in combination with IL-15 and IL-18, and this effect was abolished if IL-12 was also present. Consistent with this, different inflammatory profiles were observed in the spleen and liver of MCMV-infected mice in vivo. Although experiments in vitro cannot reproduce the complex combination of events occurring in the tissues in vivo upon infection, they defined the conditions under which PD-1 can be induced on NK cells. The correlation between the effect of the cytokines in vitro and their organ-specific expression in vivo suggests that distinct combinations of inflammatory or homeostatic cytokines could cooperate with corticosterone to induce PD-1 expression on NK cells in the spleen. The characterization of the precise inflammatory context involved in the corticosterone-induced regulation of PD-1 expression in vivo will require further investigations. For example, PD-1 was expressed on spleen NK cells in vivo despite the induction of $I 112 \mathrm{mRNA}$ in this organ. Although we did not measure IL-12 at the protein level, it is possible that different concentrations of recombinant IL-12 in vitro and in the NK cell microenvironment in vivo mediate different effects. We detected the expression of $I 118$ transcripts in the spleen and liver in vivo, but not a significant increase in the transcription of this cytokine in both organs at $44 \mathrm{~h}$ postinfection. However, as IL-18 is synthesized as an inactive precursor requiring proteolytic cleavage to produce the biologically active cytokine 29 , it is possible that expression of the mature IL-18 protein is regulated at the tissue level. In line with this, expression of mature IL-18 has been reported to be higher in the spleen than in the liver after MCMV infection 30 , providing a possible explanation for the organ-specific induction of PD-1 on spleen NK cells.

In the hematopoietic lineage, the regulation of PD-1 expression by glucocorticoids during the early stage of MCMV infection appears restricted to NK cells. It would be interesting to determine whether endogenous glucocorticoids induce PD-1 expression on various immune cells, including $\mathrm{T}$ cells, in other pathological contexts, as PD-1 expression has been reported on several immune cell subsets in other models of viral infection 28, 31, 32. PD-1 is upregulated on T cells during various conditions of activation 26. The lack of PD-1 induction on T cells early in MCMV infection observed here is expected, as Tymphocytes are activated during the later phase of the infection 17. Moreover, treatment with different combinations of inflammatory cytokines and corticosterone in vitro did not induce PD-1 expression on T cells. Thus, GR-induced PD-1 regulation is specific for NK cells. 
We showed that GR-dependent PD-1 induction on spleen NK cells was required to control the production of IFN- $\gamma$ in these cells, thereby preventing IFN- $\gamma$-dependent immunopathology in the spleen. However, the viral loads were similar in the spleens of wild-type and $\mathrm{GR}^{N c r} \mathrm{I}$-iCre mice, indicating that the downregulation of IFN- $\gamma$ production by the HPA axis did not compromise the control of viral replication. Similarly, PD-1 blockade in wild-type mice phenocopied the spleen hyperinflammation seen in $\mathrm{GR}^{N c r l-i \mathrm{Cre}}$ mice, and it did not modify the viral load. Consistent with these results, previous studies have shown that splenic NK cells exert their antiviral effects in a perforin-dependent manner, suggesting a major role for cytotoxic mechanisms in this organ 33. GR activation had no effect on the cytotoxic activity of NK cells, suggesting a decoupling between the actions of glucocorticoids on the main antiviral functions of NK cells. In the liver, IFN- $\gamma$ production by NK cells is a major mechanism for the control of MCMV replication 15, 33, 34. Despite high concentrations of corticosterone in the serum, PD-1 was not upregulated in liver NK cells, and the IFN- $\gamma$ production of these cells was unaffected. Therefore, the dampening of NK cell functions by the HPA axis does not affect the effectiveness of the anti-viral response in both the spleen and liver.

The glucocorticoid-dependent induction of PD-1 expression on NK cells in MCMV infection is a previously unrecognized mechanism by which the HPA axis suppresses immunopathology and promotes disease resistance without compromising protective immunity. It will be important to evaluate the role of this pathway in other infectious conditions or in patients with inflammatory diseases. Along this line, a population of PD-1 ${ }^{+}$ NK cells has been identified in healthy donors seropositive for HCMV 35, in HIV-1seropositive individuals 36 and in patients with Kaposi sarcoma 37, but the mechanisms of regulation of PD-1 expression on these cells remain to be addressed. It will be also interesting to analyze whether PD-1 expression on immune cells is associated with the immune dysfunctions observed in patients in other conditions in which the HPA axis is activated, such as psychological distress and tissue damage 38 .

Novel immunotherapies acting through the blockade of immune checkpoints, such as PD-1, have yielded outstanding results for advanced cancers 39,40 . In cancer patients, inflammatory reactions can be managed with immunosuppressive agents, such as corticosteroids 39. It will be important to determine in which conditions and on which cells PD-1 expression can be induced by these treatments. This analysis could make it possible to identify new pathological situations in which treatment with PD-1 antibodies could be effective. A deeper understanding of the physiological and pathological conditions in which this glucocorticoid-PD-1 pathway exerts some beneficial or detrimental effects could provide rational basis for the development of new therapeutic strategies.

\section{Methods}

\section{Mice}

Female mice on a C57BL/6 background were used in this study. Wild-type C57BL/6J mice were purchased from Janvier Labs. $\mathrm{Ncr} 1^{\mathrm{iCre} /+} \mathrm{Nr} 3 \mathrm{c} 1^{\text {LoxP/LoxP }}\left(\mathrm{GR}^{\mathrm{Ncrl}-\mathrm{iCre}}\right)$ mice were generated as previously described 6. Sex mtched $\mathrm{Ncr}^{\mathrm{iCre} /+}$ littermate (WT ${ }^{\mathrm{Ncr} l \text {-iCre }}$ ) were used as controls for $\mathrm{GR}^{N c r l-i C r e}$ mice. In some experiments in which 2 to 5 mice per 
genotype per experimental condition were analyzed, mice from several litters were used. All the mice used were bred and maintained under specific pathogen-free conditions at the Centre d'Immunophenomique (Ciphe) de Marseille and the Centre d'Immunologie de Marseille Luminy. Mice were housed under a standard $12 \mathrm{~h}: 12 \mathrm{~h}$ light-dark cycle with ad libitum access to food and water. Age-matched (7-10 weeks old) and sex-matched littermate mice were used as controls. All experiments were conducted in accordance with institutional committee recommendations (Comité d'Ethique de Marseille ${ }^{\circ} 14$ - APAFiS \#14260) and French and European guidelines for animal care.

\section{MCMV infection}

Stocks of Smith strain MCMV were generated by homogenizing salivary glands harvested from six-week-old BALB/c mice infected with $2.5 \times 10^{3} \mathrm{PFU}$ of MCMV at the age of three weeks. Mice were infected at 2 p.m., by the ip injection of $10^{3} \mathrm{PFU} / \mathrm{g}$ or $3 \times 10^{3} \mathrm{PFU} / \mathrm{g}$ (LD50) MCMV diluted in DMEM. "Non-infected" (NI) mice received DMEM only. For PD-1 blockade experiments, $250 \mu \mathrm{g}$ anti-PD-1 Ab (clone J43 or RMP1-14) or the appropriate isotype control $\mathrm{Ab}$ (Armenian Hamster IgG or IgG2a mAb clone 2A3) (all from BioXCell) were injected i.p. into mice on day 1 PI. For IFN- $\gamma$ neutralization, $500 \mu \mathrm{g}$ antiIFN $\gamma \mathrm{Ab}$ (clone XMG1.2) or Rat IgG1 (HRPN) (both from BioXCell) were injected i.p. into mice on day 1 PI. Spleens and livers were harvested after perfusion at different time points, and were processed for FACS or histology analysis, or weighed and homogenized for RNA or protein extraction. Organs were homogenized in a FastPrep-24 ${ }^{\mathrm{TM}}$ 5G homogenizer (MP Biomedicals).

\section{Viral titer and q-RT-PCR}

Organs were kept in RNAlater (Qiagen) after harvesting. RNA was extracted from organ homogenates with the RNeasy Fibrous Tissue Mini Kit (Qiagen), and reverse-transcribed with the iScript cDNA Synthesis kit (Biorad). Viral titers were determined, by qPCR, as absolute levels of the Ie1 gene (F: 5'GAGTCTGGAACCGAAACCGT3'; R:

5'GTCGCTGTTATCATTCCCCAC3', Sigma) using the SYBR Green Master Mix (Takara). For $I 115,1112$ and $I 118$ gene expression analysis, microfluidic quantitative real-time RT-PCR with the Biomark HD system (Fluidigm) was used. Briefly, pre-amplified cDNA (22 cycles) was diluted fivefold before analysis in a Flex Six IFC (Fluidigm) with Universal PCR Master Mix (Fluidigm) and ready-to-use primer and probe sets pre-developed by Applied Biosystems (TaqMan Gene Expression Assays): IL-15 (Mm00434226_m1), IL-12b (Mm00434174_m1), IL-18 (Mm00434226_m1) and GAPDH as a housekeeper (Mm99999915_g1). Ct values were calculated from the system's software (BioMark Realtime PCR Analysis; Fluidigm).

\section{In vitro splenocytes stimulation}

Total splenocytes or spleen NK cells (enriched with the NK cell isolation kit from Miltenyi Biotech) from $\mathrm{WT}^{N c r 1-\mathrm{iCre}}$ and $\mathrm{GR}^{N c r 1-\mathrm{i} C \mathrm{Cre}}$ mice were stimulated in vitro in complete culture medium (RPMI 10\% FCS, $100 \mu \mathrm{g} / \mathrm{ml}$ penicillin/streptomycin, $2 \mathrm{mM}$ l-glutamine, 1 $\mathrm{mM}$ sodium pyruvate, and $0.01 \mathrm{M}$ Hepes) with: $25 \mathrm{ng} / \mathrm{ml} \mathrm{IL-12} \mathrm{(eBiosciences),} 25 \mathrm{ng} / \mathrm{ml}$ IL-15 (Peprotech) and $20 \mathrm{ng} / \mathrm{ml}$ IL-18 (MBL) alone or in combination, or with PMA $(200 \mathrm{ng} / \mathrm{ml}$, Sigma) and ionomycin $(1 \mu \mathrm{g} / \mathrm{ml}$, Sigma). For NK1.1 stimulation 96 well plates 
(Immulon 2HB) were coated overnight at $37^{\circ} \mathrm{C}$ with $2.5 \mu \mathrm{l} /$ well anti-NK1.1 $\mathrm{Ab}$ (PK136, eBiosciences) or IgG2a isotype control before cell plating. During stimulation, 250 or 500 $\mathrm{nM}$ corticosterone (Sigma; dissolved in ethanol) or the same volume of vehicle alone were added to the medium. Cells were stimulated at $37^{\circ} \mathrm{C}$ in the presence of Golgi Stop and Golgi Plug from BD Biosciences, and then washed and stained for FACS analysis. For CD107a staining, $2.5 \mu \mathrm{l} /$ well of anti-CD107a-FITC antibody (1D4B, BD Biosciences) were added during stimulation.

\section{Cytokine levels in tissues}

We determined IFN- $\gamma$, IL-6, TNF and IL-10 protein levels in organ homogenates with cytometric bead arrays, according to the manufacturer's protocol (CBA, BD Biosciences). Results obtained in $\mathrm{pg} / \mathrm{ml}$ were converted to $\mu \mathrm{g}$ or $\mathrm{ng} / \mathrm{mg}$ of tissue considering the weight of the organ before homogenization.

\section{Serum analysis}

Blood was collected from the retro-orbital sinus of MCMV-infected mice under low-stress conditions (i.e., within $2 \mathrm{~min}$ of handling). After blood coagulation at room temperature, blood samples were centrifugated to separate the serum from the cloth. Serum samples were analyzed with the Corticosterone ELISA Kit (Enzo), according to the manufacturer's instructions, to determine corticosterone concentration, or were analysed with the cytometric bead arrays, according to the manufacturer's protocol (CBA, BD Biosciences), to determine cytokines concentration.

\section{Flow cytometry}

Single-cell suspensions were obtained from the spleen by scratching it through $70 \mu \mathrm{m}$ cell strainer, or from the liver by scratching it through $100 \mu \mathrm{m}$ cell strainer and subsequent lymphocyte isolation on a 37.5\%-67.5\% Percoll gradient. For isolation of small intestine lamina propria cells, intestines were cut longitudinally, then transversally in $2-3 \mathrm{~cm}$ pieces, thoroughly rinsed with PBS, and shaken for 30 minutes in PBS containing $10 \%$ FBS, 15mM Hepes and 5mM EDTA to remove intraepithelial and epithelial cells. Intestines were then digested with collagenase VIII (300UI/mL; Sigma) in complete RPMI for 45 minutes at $37^{\circ} \mathrm{C}$ under agitation, and lamina propria lymphocytes were isolated on a $40 \%-100 \%$ Percoll gradient. Cells were incubated with the Fc blocking antibody (2.4G2) and with a fixable blue dead-cell stain kit (Invitrogen). Surface molecules were stained with antibodies against: CD45.2 Alexa Fluor 700 (104), CD3 PE-CF594 and APC/Cy7 (145-2C11), CD19 PECF594 (1D3), NK1.1 BV510 (PK136), CD49a Alexa Fluor 647 (Ha31/8), CD11b BV510 (M1/70), MHCII BV605 (M5/114.15.2), TCRb FITC (H57-597), PD-1 BV650 (J43, and Hamster IgG2 isotype control), PDL1 PE (MIH5) from BD Biosciences; NKp46 PerCPeFluor 710 and PE/Cy7 (29A1.4), CD49b PE/Cy7 (DX5), F4/80 APC (BM8), Ly49H APC (3D10) from eBioscience; CD11c PE/Cy7 (N418), CD19 APC/Cy7 (6D5), PD-1 PE (RMP1-30, and Rat IgG2b isotype control), PDL2 PE (TY25) and Ly6G APC/Cy7 (1A8) from Biolegend. For intracellular staining, cells were fixed and permeabilized with an intracellular staining kit (eBioscience), and the following antibodies were used: anti-IFN- $\gamma$ BV421 (XMG1.2) from Biolegend; anti-granzyme B PE (GB11), anti-Ror $\gamma$ t PE-CF594 (Q31-378), anti-IL17 PE (TC11-18H10), anti-Ki67 PE (B56) from BD Biosciences, anti- 
IL-22 (JOP eBioscience, coupled to Alexa Fluor 647 with an antibody labeling kit from Life Technologies), anti-GR Alexa Fluor $488 \mathrm{XP}$ rabbit mAb (D8H2) and rabbit mAb IgG Alexa Fluor 488 XP (DA1E) from Cell Signaling Technology. Stained samples were analyzed in a BD LSRII flow cytometer (BD Biosciences). Apoptosis was detected with the FITC Annexin V Apoptosis Detection Kit I from BD Biosciences, according to the manufacturer's protocol.

Gating strategy is shown for each population in the supplementary information.

\section{Histology}

Tissues were fixed in $10 \%$ neutral buffered formalin for $24 \mathrm{~h}$, dehydrated and embedded in paraffin. Sections of $3.5 \mu \mathrm{m}$ were cut using the microtome Leica RM2245. Hematoxylineosin (H\&E) staining was effectuated automatically with Leica autostainer XL and slides were mounted with entellan and kept at room temperature. Histological slides of spleen and liver tissue were assessed by an anatomopathologist in a blinded way. For spleen inflammation grading, a score was assigned based on the severity: 0 for normal spleen, 1 for mild (multifocal pyogranulomas in marginal zones), 2 for moderate (locally coalescing pyogranulomas in marginal zones with small necrotic foci), 3 for marked (large and coalescing pyogranulomas throughout the splenic parenchyma with extensive necrotic foci, PALS are preserved), and 4 for severe (extensive necrotic and pyogranulomatous foci, PALS are partially replaced by necrotic and granulomatous inflammation). For liver inflammation grading, a score was assigned based on the severity: 0 for normal, 1 for mild (multifocal pyogranulomatous hepatitis with scattered single necrotic hepatocytes), 2 for moderate (multifocal to coalescing necrotic and pyogranulomatous hepatitis with intranuclear inclusions in hepatocytes), and 3 for marked (coalescing necrotic and pyogranulomatous hepatitis with intranuclear inclusions in hepatocytes). For the morphometric assessment of periarteriolar lymphoid sheath (PALS), the area of PALS and the total area of spleen section were measured using the CaseViewer software from 3Dhistech. Analysis was done on pictures taken with a Nikon Eclipse Ci microscope, on random cross sections of the spleens.

\section{Cell sorting and mRNAseq analysis}

Splenocytes (after NK cell enrichment with the mouse NK Cell Isolation Kit II, Miltenyi Biotec) and liver lymphocytes were pooled from three mice for each genotype. A FACS Aria III (BD Biosciences) was used to sort approximately $5 \times 10^{5} \mathrm{NK}$ cells from the spleen and liver and $5 \times 10^{4}$ liver-resident ILC1s. Cells were sorted directly in RLT lysis buffer (Qiagen). Total RNA was prepared from purified ILC populations with an RNeasy Micro Kit (Qiagen). Three biological replicates were generated for all samples except for the $\mathrm{GR}^{\text {Ncr } 1 \text {-iCre }}$ liver ILC1s sample (two biological replicates). Preamplification was performed with the SMART-Seq ${ }^{\circledR}$ v4 Ultra ${ }^{\circledR}$ Low-Input RNA Kit (Clontech). The DNA libraries were generated by double-indexing with the Nextera XT DNA Kit (Illumina) and RNA sequencing was performed with a NextSeq 500 (Illumina; paired-end reads $2 \times 75$ with $30 \mathrm{M}$ reads per sample). The fastq files were assessed with the fastqc program and trimming was performed with Trimmomatics, to remove potential molecular barcodes, Illumina adapters and low-quality reads. Alignment was performed with two algorithms: firstly, with bowtie2 vs GRCm38 ensemble transcriptome resulting in a BAM that could be processed with the 
molecular index provided by the kit vendor, and, secondly, with HiSat2 over the GRCm38 genome, for the detection of novel junction regions. Duplicates were detected and removed by MarkDuplicates from picard tools, and the number of reads mapped to each gene was determined with featureCounts v1.5.2. Normalization and differential analysis were performed with DESeq2 v1.16.1. HalioDx (Marseille, France) processed the RNA samples and bioinformatics analyses were performed by the CIML platform.

\section{Statistical analysis}

No sample size calculation was performed, but a reasonable sample size was chosen to ensure adequate reproducibility of results and was based on our previous studies. Mice were assigned to experimental groups according to sex and age. Statistical analysis was performed with Graphpad Prism 7 Software. Normality was tested with the Shapiro-Wilk test. Unpaired two-tailed Student's t-tests were used if the data followed a Gaussian distribution with similar variances. Mann-Whitney $U$ tests were performed if this was not the case. One-way ANOVA was used for multigroup comparisons. Differences in survival were evaluated with Mantel-Cox tests. Differences were considered significant for P values less than 0.05 .

\section{Reporting Summary}

Further information on experimental design is available in the Nature Research Reporting Summary linked to this article.

\section{Supplementary Material}

Refer to Web version on PubMed Central for supplementary material.

\section{Acknowledgments}

We thank F. Tronche for providing the $N_{r} 3 c 1^{L o x P} / L o x P$ mice. We thank J. Galluso and P. Morganti for mouse breeding and genotyping and G. Bessou for providing the plasmid containing the iel gene. We thank the Centre d'Immunologie de Marseille-Luminy (CIML) mouse house and core cytometry facilities. This project received funding from the European Research Council (ERC) under the European Union's Horizon 2020 research and innovation program, under grant agreement No. 648768; from the Agence Nationnale de la Recherche (ANR) (No. ANR-14-CE14-0009-01) and from the ARC foundation (No. PGA120140200817). This work was also supported by institutional grants from INSERM, CNRS, Aix-Marseille University and Marseille-Immunopole to the CIML.

\section{References}

1. Medzhitov R, Schneider DS, Soares MP. Disease tolerance as a defense strategy. Science. 2012; 335:936-941. [PubMed: 22363001]

2. Webster JI, Tonelli L, Sternberg EM. Neuroendocrine regulation of immunity. Annu Rev Immunol. 2002; 20:125-163. [PubMed: 11861600]

3. Irwin MR, Cole SW. Reciprocal regulation of the neural and innate immune systems. Nat Rev Immunol. 2011; 11:625-632. [PubMed: 21818124]

4. Cain DW, Cidlowski JA. Immune regulation by glucocorticoids. Nat Rev Immunol. 2017; 17:233247. [PubMed: 28192415]

5. Weikum ER, Knuesel MT, Ortlund EA, Yamamoto KR. Glucocorticoid receptor control of transcription: precision and plasticity via allostery. Nat Rev Mol Cell Biol. 2017; 18:159-174. [PubMed: 28053348]

6. Quatrini L, et al. Host resistance to endotoxic shock requires the neuroendocrine regulation of group 1 innate lymphoid cells. J Exp Med. 2017 
7. Bhattacharyya S, Brown DE, Brewer JA, Vogt SK, Muglia LJ. Macrophage glucocorticoid receptors regulate Toll-like receptor 4-mediated inflammatory responses by selective inhibition of p38 MAP kinase. Blood. 2007; 109:4313-4319. [PubMed: 17255352]

8. Li CC, Munitic I, Mittelstadt PR, Castro E, Ashwell JD. Suppression of Dendritic Cell-Derived IL-12 by Endogenous Glucocorticoids Is Protective in LPS-Induced Sepsis. PLoS Biol. 2015; 13:e1002269. [PubMed: 26440998]

9. Kleiman A, et al. Glucocorticoid receptor dimerization is required for survival in septic shock via suppression of interleukin-1 in macrophages. FASEB J. 2012; 26:722-729. [PubMed: 22042221]

10. Jamieson AM, Yu S, Annicelli CH, Medzhitov R. Influenza virus-induced glucocorticoids compromise innate host defense against a secondary bacterial infection. Cell Host Microbe. 2010; 7:103-114. [PubMed: 20159617]

11. Ruzek MC, Miller AH, Opal SM, Pearce BD, Biron CA. Characterization of early cytokine responses and an interleukin (IL)-6-dependent pathway of endogenous glucocorticoid induction during murine cytomegalovirus infection. J Exp Med. 1997; 185:1185-1192. [PubMed: 9104805]

12. Ruzek MC, Pearce BD, Miller AH, Biron CA. Endogenous glucocorticoids protect against cytokine-mediated lethality during viral infection. J Immunol. 1999; 162:3527-3533. [PubMed: 10092810]

13. Rhen T, Cidlowski JA. Antiinflammatory action of glucocorticoids--new mechanisms for old drugs. N Engl J Med. 2005; 353:1711-1723. [PubMed: 16236742]

14. Orange JS, Biron CA. Characterization of early IL-12, IFN-alphabeta, and TNF effects on antiviral state and NK cell responses during murine cytomegalovirus infection. J Immunol. 1996; 156:4746-4756. [PubMed: 8648121]

15. Orange JS, Biron CA. An absolute and restricted requirement for IL-12 in natural killer cell IFNgamma production and antiviral defense. Studies of natural killer and T cell responses in contrasting viral infections. J Immunol. 1996; 156:1138-1142. [PubMed: 8557990]

16. Weizman OE, et al. ILC1 Confer Early Host Protection at Initial Sites of Viral Infection. Cell. 2017

17. Krmpotic A, Bubic I, Polic B, Lucin P, Jonjic S. Pathogenesis of murine cytomegalovirus infection. Microbes Infect. 2003; 5:1263-1277. [PubMed: 14623023]

18. Loh J, Chu DT, O'Guin AK, Yokoyama WM, Virgin HWt. Natural killer cells utilize both perforin and gamma interferon to regulate murine cytomegalovirus infection in the spleen and liver. J Virol. 2005; 79:661-667. [PubMed: 15596864]

19. Biron CA, Tarrio ML. Immunoregulatory cytokine networks: 60 years of learning from murine cytomegalovirus. Med Microbiol Immunol. 2015; 204:345-354. [PubMed: 25850988]

20. Tronche F, et al. Disruption of the glucocorticoid receptor gene in the nervous system results in reduced anxiety. Nat Genet. 1999; 23:99-103. [PubMed: 10471508]

21. Narni-Mancinelli E, et al. Fate mapping analysis of lymphoid cells expressing the NKp46 cell surface receptor. Proc Natl Acad Sci U S A. 2011; 108:18324-18329. [PubMed: 22021440]

22. Spits $\mathrm{H}$, et al. Innate lymphoid cells--a proposal for uniform nomenclature. Nat Rev Immunol. 2013; 13:145-149. [PubMed: 23348417]

23. Ayroldi E, Riccardi C. Glucocorticoid-induced leucine zipper (GILZ): a new important mediator of glucocorticoid action. FASEB J. 2009; 23:3649-3658. [PubMed: 19567371]

24. Delfino DV, Agostini M, Spinicelli S, Vito P, Riccardi C. Decrease of Bcl-xL and augmentation of thymocyte apoptosis in GILZ overexpressing transgenic mice. Blood. 2004; 104:4134-4141. [PubMed: 15319285]

25. Schmidt S, et al. Glucocorticoid-induced apoptosis and glucocorticoid resistance: molecular mechanisms and clinical relevance. Cell Death Differ. 2004; 11(Suppl 1):S45-55. [PubMed: 15243581]

26. Sharpe AH, Pauken KE. The diverse functions of the PD1 inhibitory pathway. Nat Rev Immunol. 2017

27. Arase H, Mocarski ES, Campbell AE, Hill AB, Lanier LL. Direct recognition of cytomegalovirus by activating and inhibitory NK cell receptors. Science. 2002; 296:1323-1326. [PubMed: 11950999]

28. Chen Y, et al. Programmed death (PD)-1-deficient mice are extremely sensitive to murine hepatitis virus strain-3 (MHV-3) infection. PLoS Pathog. 2011; 7:e1001347. [PubMed: 21750671] 
29. Okamura H, Tsutsui H, Kashiwamura S, Yoshimoto T, Nakanishi K. Interleukin-18: a novel cytokine that augments both innate and acquired immunity. Adv Immunol. 1998; 70:281-312. [PubMed: 9755340]

30. Pien GC, Satoskar AR, Takeda K, Akira S, Biron CA. Cutting edge: selective IL-18 requirements for induction of compartmental IFN-gamma responses during viral infection. J Immunol. 2000; 165:4787-4791. [PubMed: 11046000]

31. Frebel $\mathrm{H}$, et al. Programmed death 1 protects from fatal circulatory failure during systemic virus infection of mice. J Exp Med. 2012; 209:2485-2499. [PubMed: 23230000]

32. Erickson JJ, et al. Viral acute lower respiratory infections impair CD8+ T cells through PD-1. J Clin Invest. 2012; 122:2967-2982. [PubMed: 22797302]

33. Tay CH, Welsh RM. Distinct organ-dependent mechanisms for the control of murine cytomegalovirus infection by natural killer cells. J Virol. 1997; 71:267-275. [PubMed: 8985346]

34. Orange JS, Wang B, Terhorst C, Biron CA. Requirement for natural killer cell-produced interferon gamma in defense against murine cytomegalovirus infection and enhancement of this defense pathway by interleukin 12 administration. J Exp Med. 1995; 182:1045-1056. [PubMed: 7561678]

35. Della Chiesa M, et al. Features of Memory-Like and PD-1(+) Human NK Cell Subsets. Front Immunol. 2016; 7:351. [PubMed: 27683578]

36. Norris S, et al. PD-1 expression on natural killer cells and CD8(+) T cells during chronic HIV-1 infection. Viral Immunol. 2012; 25:329-332. [PubMed: 22742708]

37. Beldi-Ferchiou A, et al. PD-1 mediates functional exhaustion of activated NK cells in patients with Kaposi sarcoma. Oncotarget. 2016; 7:72961-72977. [PubMed: 27662664]

38. Glaser R, Kiecolt-Glaser JK. Stress-induced immune dysfunction: implications for health. Nat Rev Immunol. 2005; 5:243-251. [PubMed: 15738954]

39. Sharma P, Allison JP. Immune checkpoint targeting in cancer therapy: toward combination strategies with curative potential. Cell. 2015; 161:205-214. [PubMed: 25860605]

40. Sharma P, Allison JP. The future of immune checkpoint therapy. Science. 2015; 348:56-61. [PubMed: 25838373] 

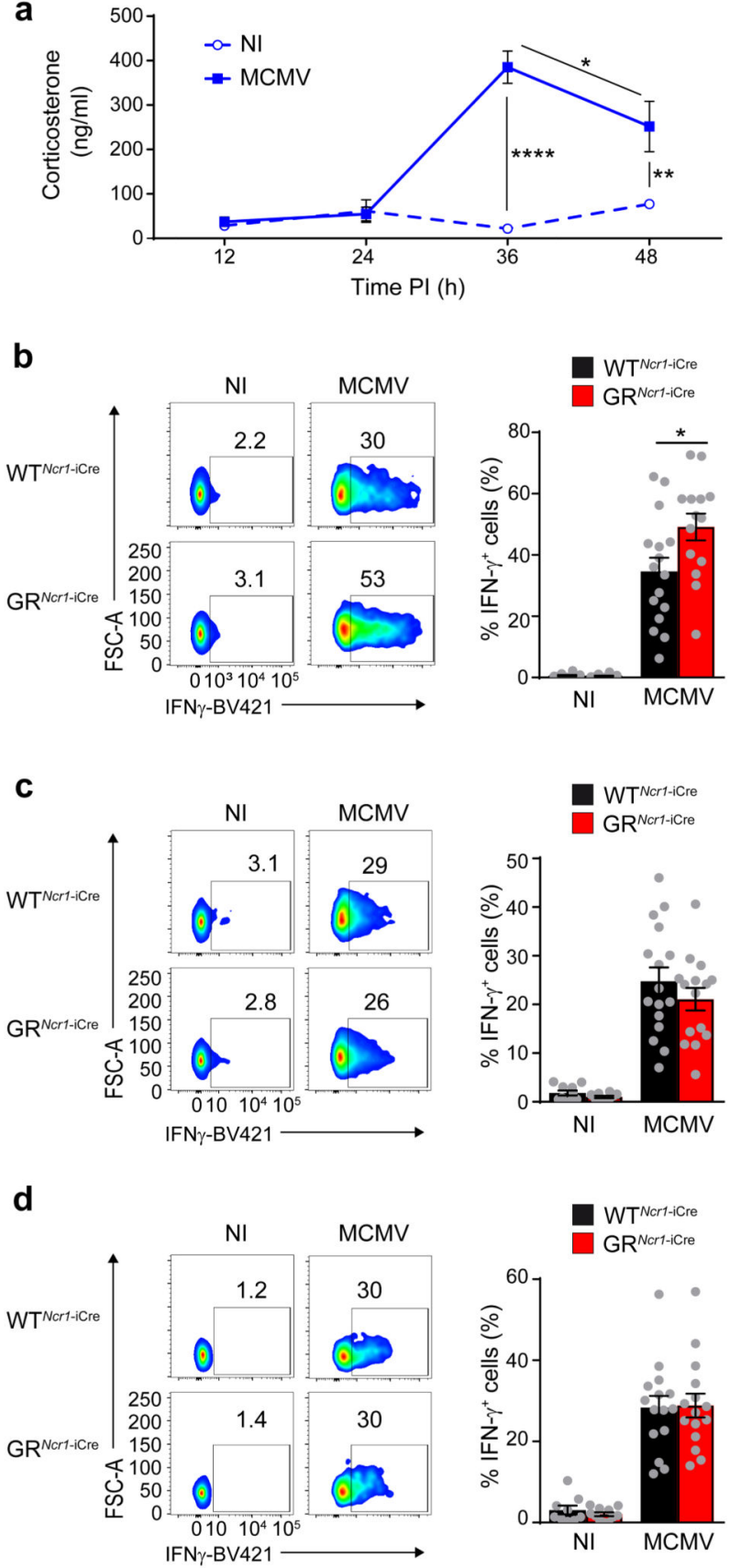

Figure 1. Organ-specific glucocorticoid regulation of NCR1+ ILCs IFN- $\gamma$ production in spleen and liver upon MCMV infection.

a, ELISA showing corticosterone concentration in the serum of WT mice receiving injections of MCMV ( $n=5)$ or DMEM (NI, non-infected) $(n=5)$, pool of 2 experiments. Means \pm s.e.m., $* P<0.05$, $* * P<0.01$, $* * * * P<0.0001$, one-way ANOVA. b-d, FACS analysis of intracellular IFN- $\gamma$, assessed directly ex vivo without in vitro re-stimulation. Frequencies of IFN- $\gamma$-producing spleen NK cells (b, $n=10$ NI, $n=16 \mathrm{WT}^{N c r 1-\mathrm{iCre}} \mathrm{MCMV}$, or $n=15$ $\mathrm{GR}^{\text {Ncrl-iCre }}$ MCMV mice; pool of 5 experiments), liver NK cells (c, $n=10$ NI, $n=16$ 
$\mathrm{WT}^{N c r l-\mathrm{iCre}} \mathrm{MCMV}$, or $n=15 \mathrm{GR}^{N c r l \text {-iCre }}$ MCMV mice; pool of 5 experiments) and liver ILC1s (d, $n=9 \mathrm{WT}^{N c r 1 \text {-iCre }} \mathrm{NI}, n=11 \mathrm{GR}^{N c r l-\text {-iCre }} \mathrm{NI}, n=16 \mathrm{WT}^{N c r l \text {-iCre }}$ MCMV, or $n=15$ $\mathrm{GR}^{\text {Ncr } 1 \text {-iCre }}$ MCMV mice; pool of 5 experiments) $44 \mathrm{~h}$ post-infection (PI) are shown as means \pm s.e.m. and representative FACS plots. ${ }^{*} P<0.05$, two-tailed Student's $t$-test. Every symbol in b-d represents a single mouse. 


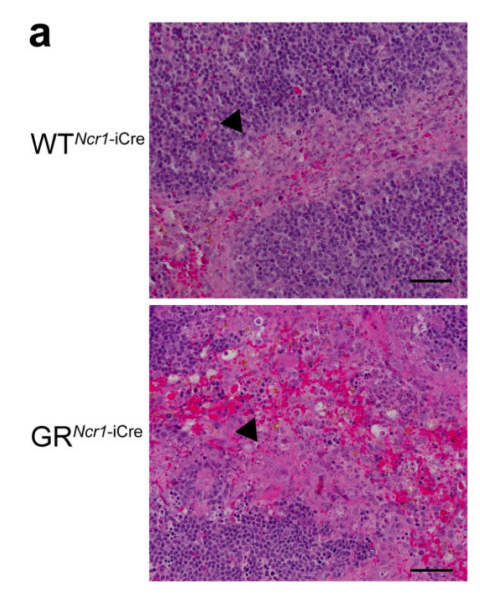

b
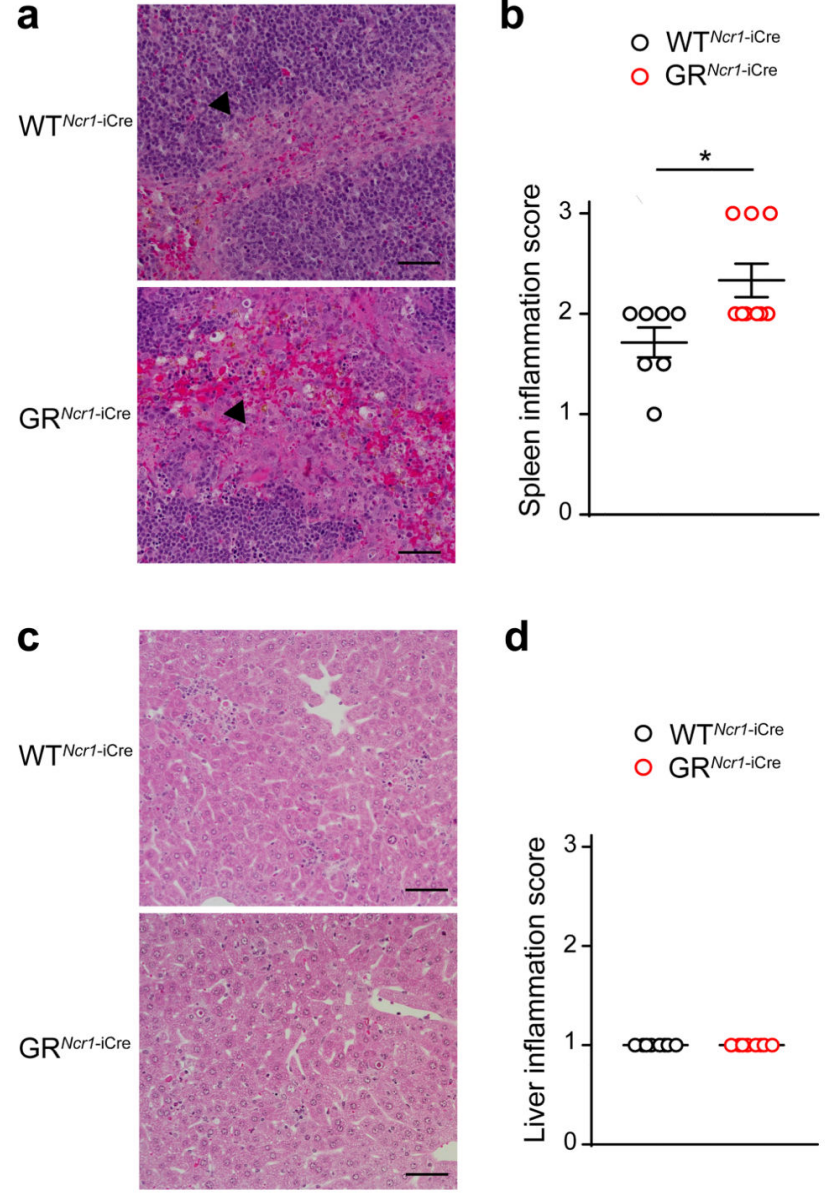

d

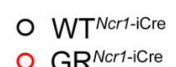

O GR Nor1-icre $^{-1}$

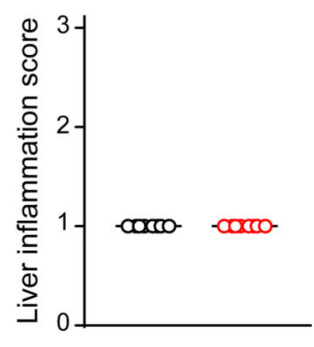

e

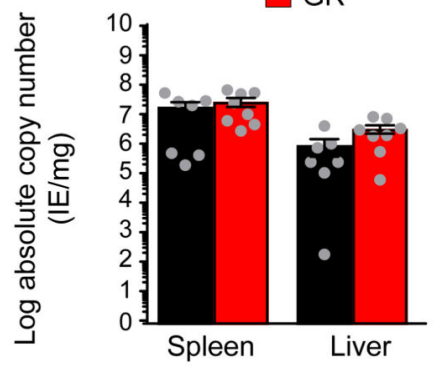

Figure 2. GR ${ }^{N c r 1-i C r e}$ mice display more marked inflammation in the spleen than $\mathrm{WT}^{N c r 1-\mathrm{iCre}}$ mice but their viral titer is unaffected.

Histology (H\&E staining) of spleen (a) and liver (c) 44 h PI. Representative images of 8 mice per genotype from 2 experiments are shown. The arrowheads in $\mathbf{a}$ indicate the inflammation area in the marginal zone. The bar represents $50 \mu \mathrm{m}$. b and $\mathbf{d}$, histopathological quantification of spleen (b) and liver (d) inflammation: 1=mild, $2=$ moderate and $3=$ marked. $n=7\left(\mathrm{WT}^{N c r 1 \text {-iCre }}\right)$ and $n=9\left(\mathrm{GR}^{N c r 1 \text {-iCre }}\right)$ mice, pool of 2 experiments. Means \pm s.e.m., ${ }^{*} P<0.05$, two-tailed Mann-Whitney test. e, qPCR absolute 
quantification of viral titer at 44 h PI. $n=7$ (WT $\left.{ }^{N c r l-\mathrm{iCre}}\right)$ and $n=8\left(\mathrm{GR}^{N c r l-\mathrm{iCre}}\right)$ mice; pool of 2 experiments. Means \pm s.e.m.. Every symbol in $\mathbf{b}, \mathbf{d}$ and $\mathbf{e}$ represents a single mouse. 
a

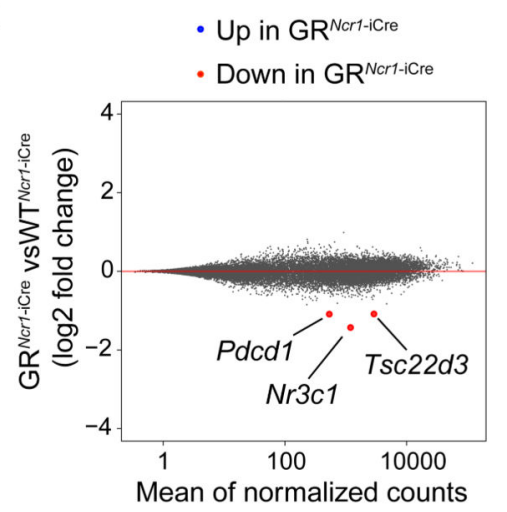

C

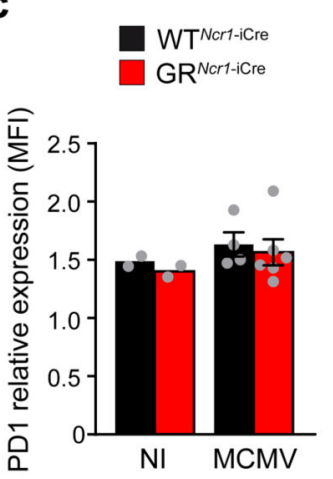

b
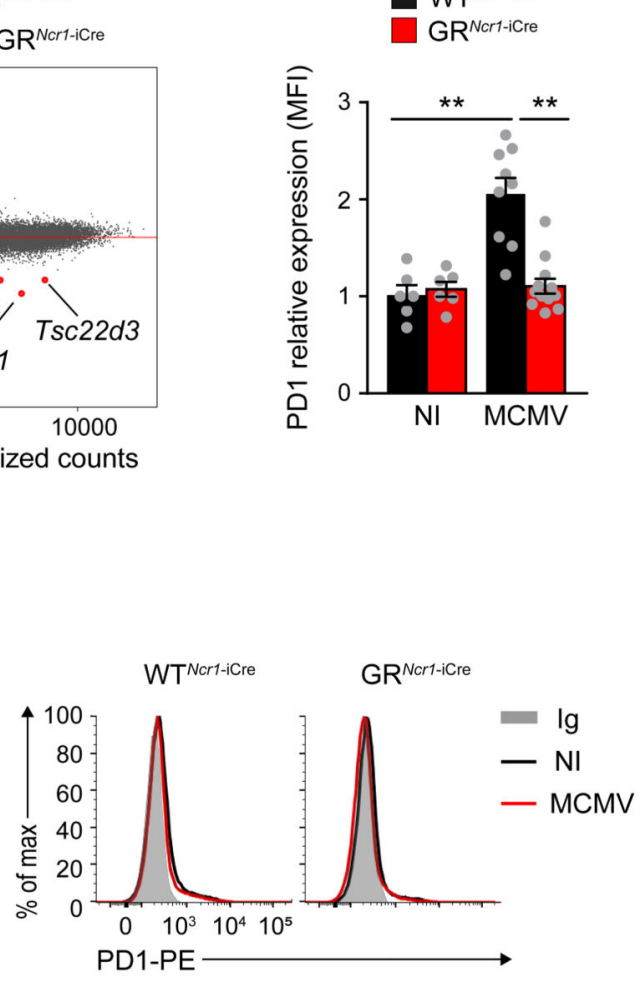

d

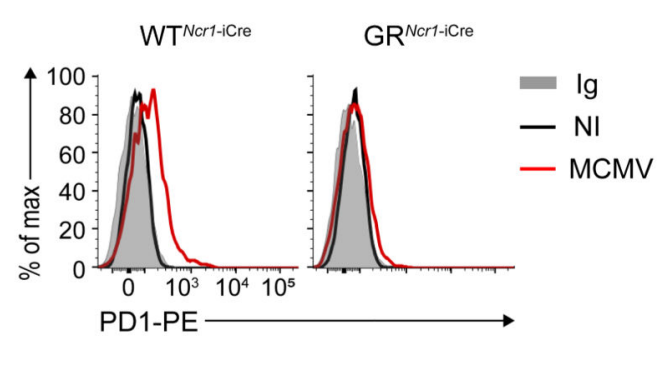

WT $T^{\text {Ner-THCre }}$

e

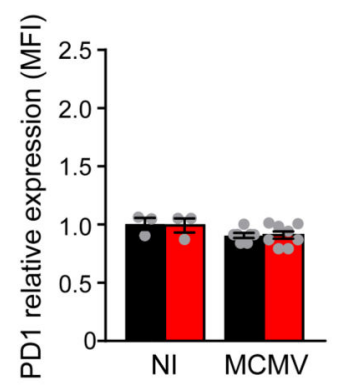

WT $T^{\text {Nar1-icre }}$

$\mathrm{GR}^{\mathrm{Nar1-iCre}}$

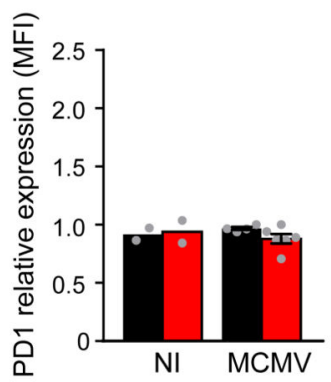

Figure 3. MCMV infection induces glucocorticoid-dependent PD-1 expression in spleen NK cells. a. RNA Seq analysis. MA plot of genes differentially expressed between spleen NK cells sorted from $\mathrm{GR}^{N c r 1-\mathrm{iCre}}$ and $\mathrm{WT}^{N c r l-\mathrm{iCre}} 1$ mice $44 \mathrm{~h}$ PI. Genes with significantly lower (red) levels of expression in GR-deficient NK cells are highlighted (absolute $\log _{2}$ fold-change $>1$ and $P<0.05)$. $n=3$ samples $\mathrm{GR}^{N c r l-\mathrm{iCre}}$ versus $3 \mathrm{WT}^{N c r l-\mathrm{iCre}}$ samples. b-e FACS analysis of cell surface PD-1 expression in spleen NK cells (b, $n=6 \mathrm{NI}, n=9 \mathrm{WT}^{\text {Ncr } 1 \text {-iCre }} \mathrm{MCMV}$ and $n=12 \mathrm{GR}^{N c r l-\mathrm{iCre}} \mathrm{MCMV}$ mice; pool of 3 experiments), spleen T cells (c, $n=2 \mathrm{NI}, n=4$ $\mathrm{WT}^{N c r 1 \text {-iCre }}$ MCMV and $n=6 \mathrm{GR}^{N c r 1 \text {-iCre }}$ MCMV mice; pool of 2 experiments), liver NK cells (d, $n=3 \mathrm{NI}, n=7 \mathrm{WT}^{N c r l-\mathrm{iCre}}$ MCMV and $n=8 \mathrm{GR}^{N c r l-\text { iCre }}$ MCMV mice; pool of 2 experiments) and liver ILC1s (e, $n=2 \mathrm{NI}, n=4 \mathrm{WT}^{N c r l-\text { iCre }} \mathrm{MCMV}$ and $n=6 \mathrm{GR}^{N c r l-\mathrm{iCre}}$ MCMV mice; pool of 2 experiments) 44h PI shown as MFI (mean fluorescence intensity) ratio relative to isotype control. Means \pm s.e.m., $* * P<0.01$, one-way ANOVA. Similar results were obtained using two different anti-PD-1 antibodies (J43 and RMP1-30). Every symbol in b-e represents a single mouse. 
b
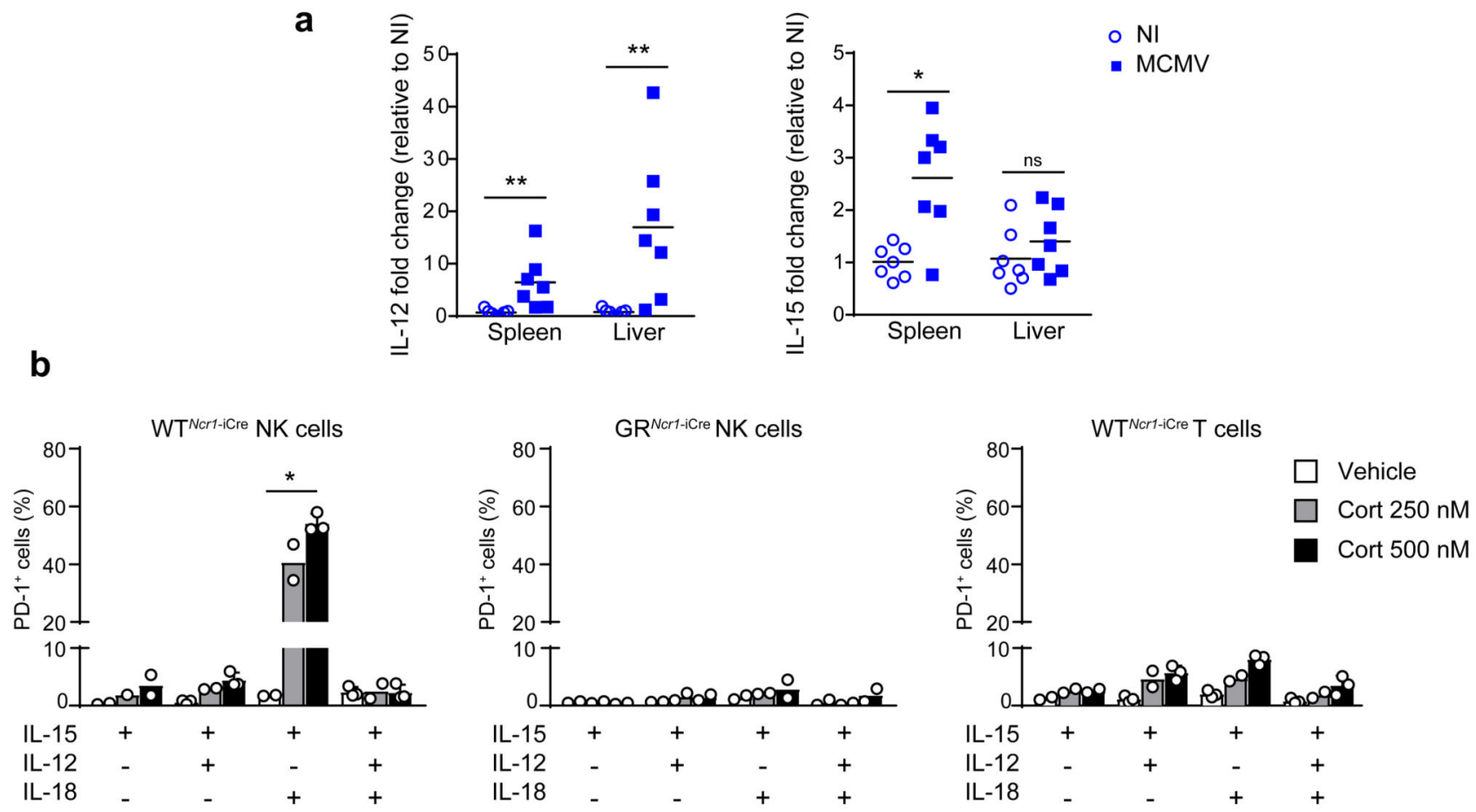

C

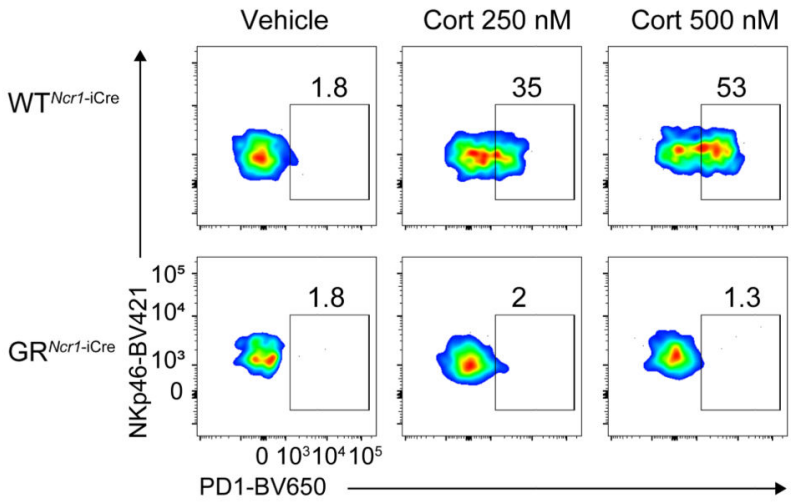

d
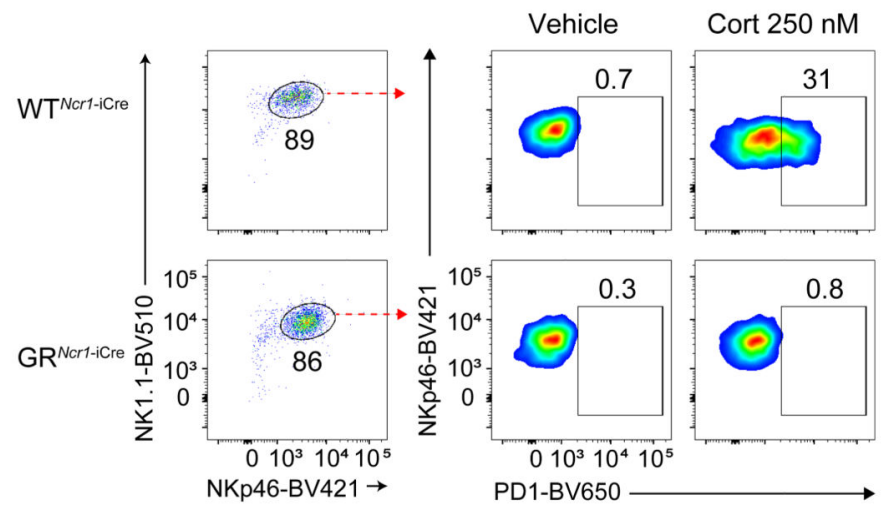

- MCMV 
$* P<0.05$, one-way ANOVA. Means \pm s.d. and representative FACS plots of IL- $15+$ IL-18 stimulation (c and $\mathbf{d}$ ) are shown. Every symbol in $\mathbf{b}$ represents a single experiment. 
a

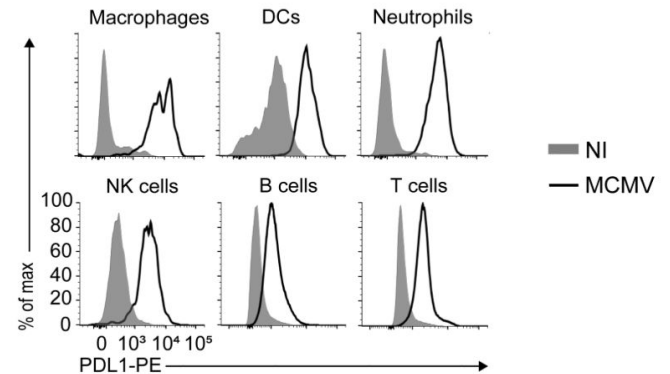

b
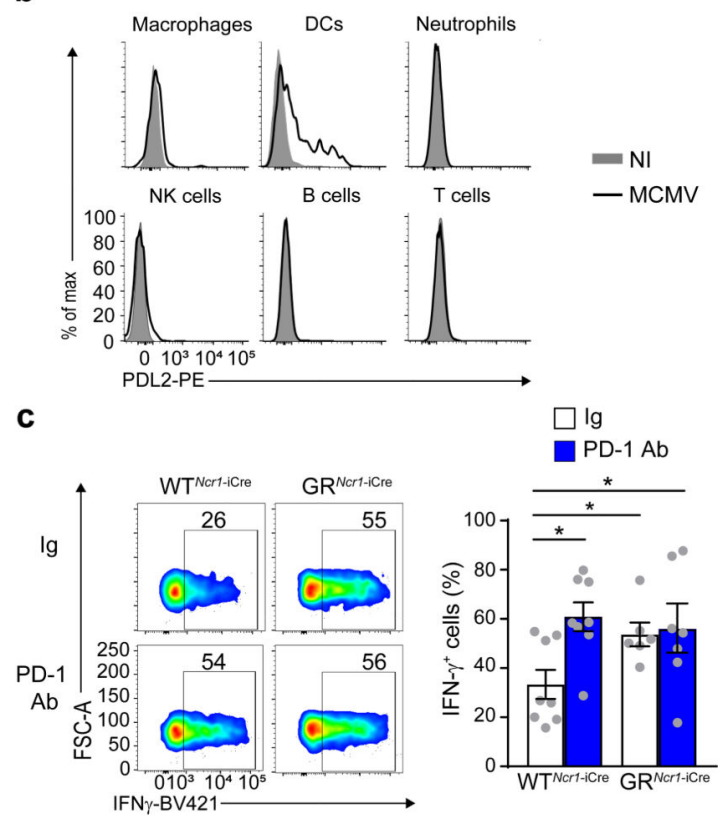

d
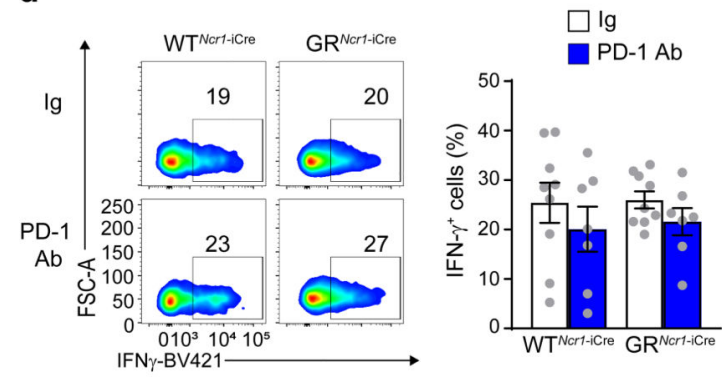

Figure 5. MCMV-induced IFN- $\gamma$ production by spleen NK cells is regulated by a glucocorticoidPD-1 axis.

$\mathbf{a}$ and b, FACS analysis of cell surface PDL1 (a) and PDL2 (b) expression $44 \mathrm{~h}$ PI by

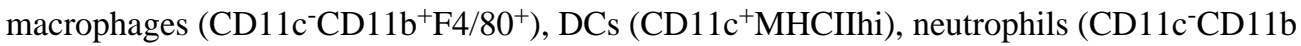
$\left.{ }^{+} \mathrm{Ly}_{6 \mathrm{G}^{+}}\right)$, NK cells $\left(\mathrm{NK} 1.1^{+} \mathrm{NKp} 46^{+} \mathrm{CD} 49 \mathrm{~b}^{+}\right)$, B cells $\left(\mathrm{CD} 19^{+}\right)$and $\mathrm{T}$ cells $\left(\mathrm{CD}^{+} \mathrm{NK} 1.1^{-}\right)$ in the spleen ( $n=2$ independent experiments). $\mathbf{c}$ and $\mathbf{d}$, FACS analysis of intracellular IFN- $\gamma$, assessed directly ex vivo without in vitro restimulation. Frequency of IFN- $\gamma$-producing NK cells in the spleen (c) and liver (d) $44 \mathrm{~h} \mathrm{PI}$, following treatment with an anti-PD-1 antibody 
or an isotype control antibody (Ig). c, $n=8$ (WT ${ }^{N c r 1-\mathrm{iCre}} \operatorname{Ig}$ and PD-1 Ab), $n=6\left(\mathrm{GR}^{N c r 1-\mathrm{iCre}}\right.$ $\mathrm{Ig}$ ), and $n=7$ (GR ${ }^{N c r l}$-iCre PD-1 Ab) mice; pool of 4 experiments. $\mathbf{d}$, $n=9$ (Ig) and $n=7$ (PD-1 $\mathrm{Ab}$ ) mice; pool of 4 experiments. Means \pm s.e.m. and representative FACS plots. $* P<0.05$, one-way ANOVA. Every symbol represents a single mouse. 
a

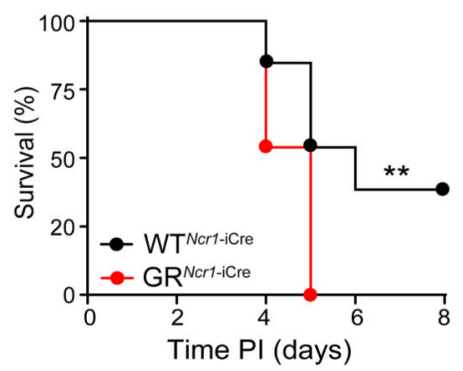

b

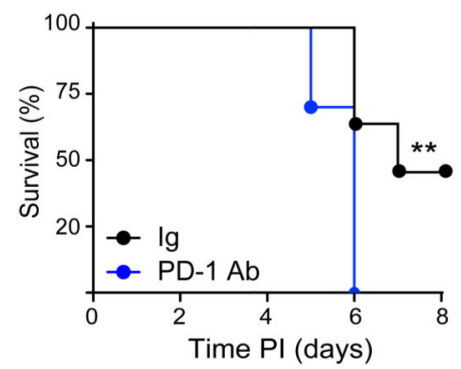

C

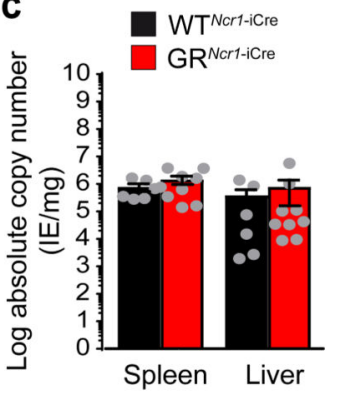

d

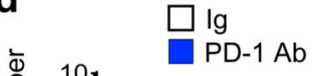

e

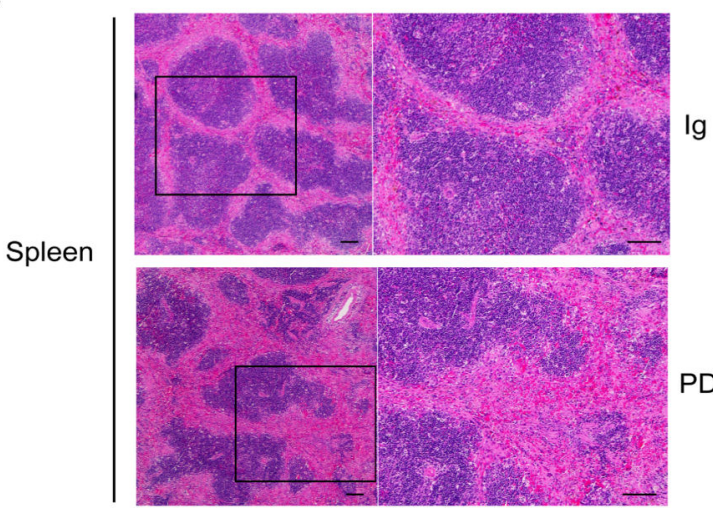

$\lg$

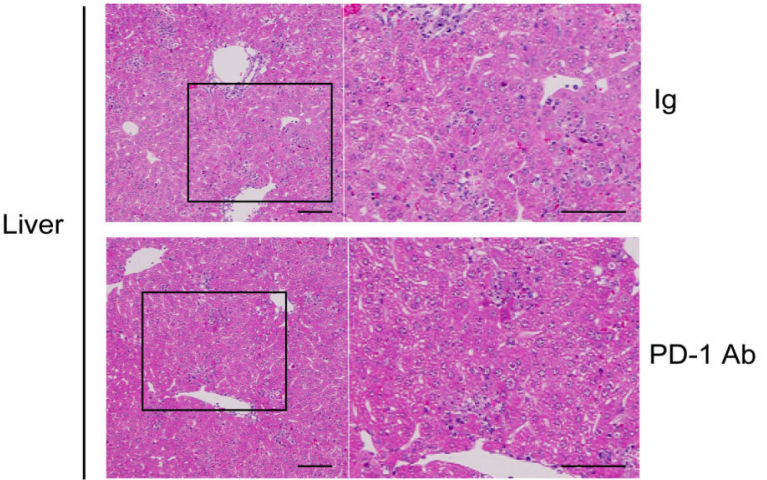

f
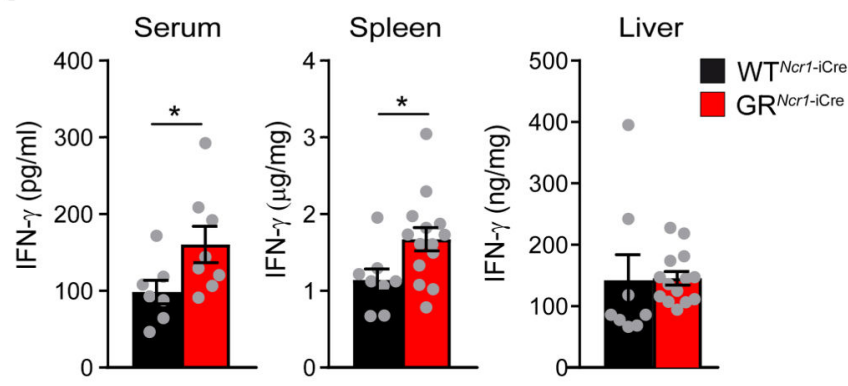

g
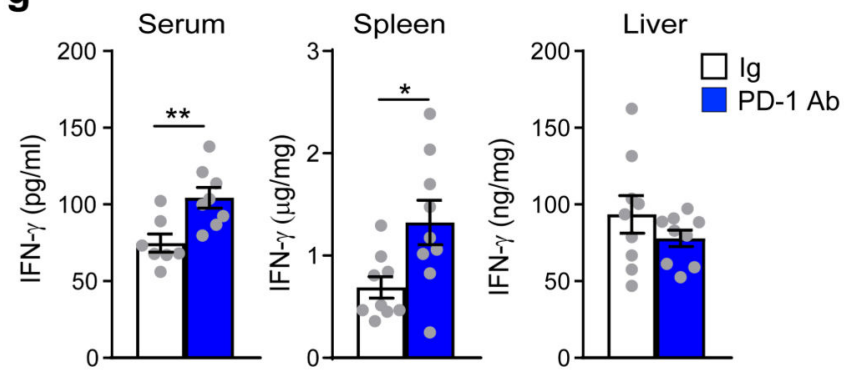

Figure 6. The glucocorticoid-PD-1 regulatory pathway is required for protection against MCMV infection.

(a) Survival curve for mice infected with MCMV ( $n=13$, pool of 3 experiments), and (b) receiving injections of anti-PD-1 or an isotype control antibody (Ig). $n=11$ (Ig) and $n=10$ (PD-1 Ab), pool of 2 experiments. ${ }^{* *} P<0.01$, two-tailed log-rank Mantel-Cox test. $\mathbf{c}$ and $\mathbf{d}$, qPCR absolute quantification of viral titer 3 days PI. c, $n=6$ (WT ${ }^{N c r l-\mathrm{iCre}}$ ) and $n=9$ (GR $^{N c r l-\text { iCre }}$ ) mice, pool of 2 experiments. d, $n=9$ (Ig) and $n=10$ (PD-1 Ab) mice, pool of 2 experiments. e, Histology (H\&E staining) of spleen and liver 3 days PI. For each panel, a higher magnification of the corresponding areas in the black boxes is shown. The bar represents $100 \mu \mathrm{m}$. Similar results were obtained with two different anti-PD-1 antibody clones (J43 and RMP1-14). $n=4$ (Ig) and $n=5$ (PD-1 Ab) mice. $\mathbf{f}$ and $\mathbf{g}$, cytometric bead array measurement of IFN- $\gamma$ concentration 3 days PI. f, $n=7$ (serum WT ${ }^{N c r} 1$-iCre),$n=8$ (serum $\mathrm{GR}^{N c r} 1$-iCre and $\mathrm{WT}^{N c r l-\mathrm{iCre}}$ spleen and liver), and $n=14\left(\mathrm{GR}^{N c r l-\mathrm{iCre}}\right.$ spleen and 
liver) mice; pool of 2 experiments (serum) and pool of 3 experiments (spleen and liver). $* \mathrm{P}<0.05$, two-tailed Mann-Whitney test (serum) and two-tailed Student's t-test (spleen). g, $n=7$ (Ig serum), $n=8$ (PD-1 Ab serum), and $n=9$ (spleen and liver) mice; pool of 2 experiments. ${ }^{*} \mathrm{P}<0.01$, two-tailed Mann-Whitney test (serum) and $* \mathrm{P}<0.05$, two-tailed Student's t-test (spleen). Means \pm s.e.m. are shown for $\mathbf{c}, \mathbf{d}, \mathbf{f}$ and $\mathbf{g}$. Each symbol represents a single mouse. 
a
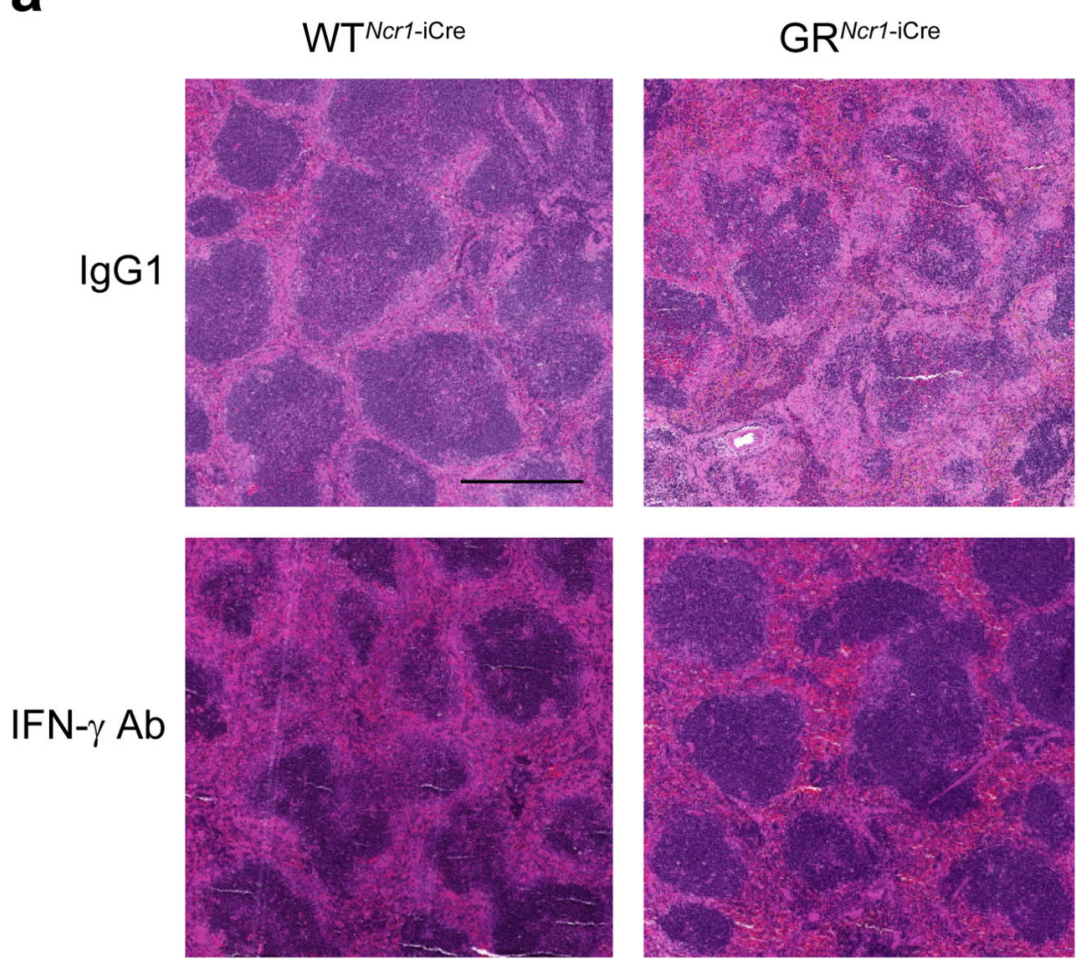

b

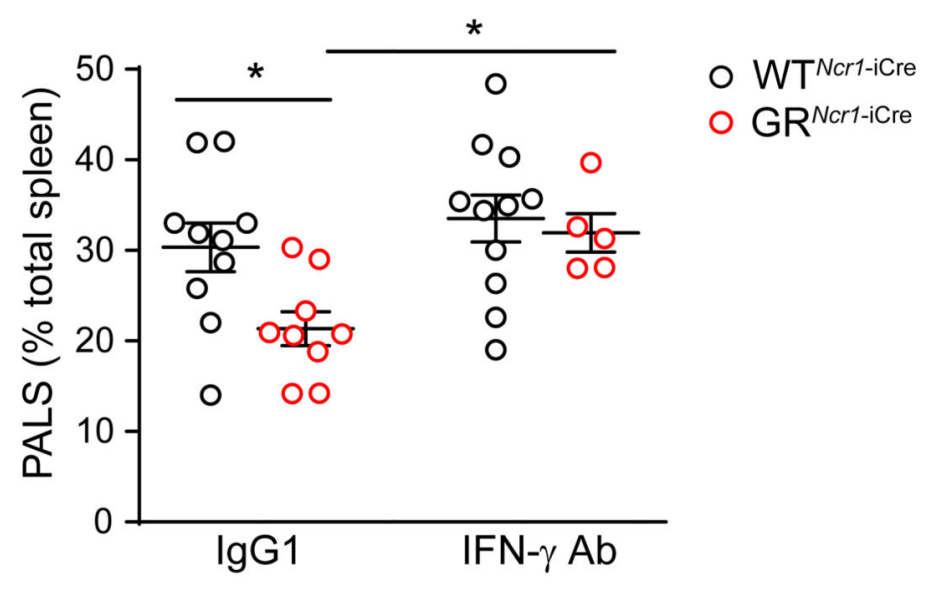

Figure 7. IFN- $\gamma$ neutralization prevents spleen immunopathology in $\mathbf{G R}^{N c r 1-\mathrm{iCre}}$ mice. Histology (H\&E staining) of the spleen 3 days PI. Mice were infected with the LD50 of MCMV and injected on day 1 PI with anti-IFN $\gamma$ or IgG1 isotype control antibodies. The bar represents $500 \mu \mathrm{m}$. b, histopathological quantification of spleen inflammation: the area covered by PALS was measured and expressed as percentage of the total area of the spleen section. $n=10$ (WT $\left.{ }^{N c r l-\mathrm{iCre}} \operatorname{IgG} 1\right), n=9\left(\mathrm{GR}^{N c r l \text {-iCre }} \mathrm{IgG} 1\right), n=11$ (WT $\left.{ }^{N c r l-\mathrm{iCre}} \mathrm{IFN}-\gamma \mathrm{Ab}\right)$, 
and $n=5\left(\mathrm{GR}^{N c r} 1\right.$-iCre $\left.\mathrm{IFN}-\gamma \mathrm{Ab}\right)$ mice; pool of 2 experiments. $* P<0.05$, one-way ANOVA, $n s=$ not significant. Means \pm s.e.m.. Each symbol represents a single mouse. 